\title{
ERROR ESTIMATES FOR THE NUMERICAL APPROXIMATION OF SEMILINEAR ELLIPTIC CONTROL PROBLEMS WITH FINITELY MANY STATE CONSTRAINTS*
}

\author{
EDUARDO CASAS ${ }^{1}$
}

\begin{abstract}
The goal of this paper is to derive some error estimates for the numerical discretization of some optimal control problems governed by semilinear elliptic equations with bound constraints on the control and a finitely number of equality and inequality state constraints. We prove some error estimates for the optimal controls in the $L^{\infty}$ norm and we also obtain error estimates for the Lagrange multipliers associated to the state constraints as well as for the optimal states and optimal adjoint states.
\end{abstract}

Mathematics Subject Classification. 49J20, 49K20, 49M05, 65K10.

Received January 14, 2002.

\section{INTRODUCTION}

In this paper we study an optimal control problem governed by a semilinear elliptic equation, the control being distributed in $\Omega$. Bound constraints on the control and finitely many equality and inequality state constraints are included in the formulation of the problem. Integral constraints on the state falls into this formulation. The aim is to consider the numerical approximation of this problem by using finite element methods. We prove that under certain assumptions the discrete problems have optimal solutions. We also prove that these solutions converge uniformly towards solutions of the infinity dimensional problem. By making a qualification assumption we deduce the existence of Lagrange multipliers associated with the state constraints for the continuous and discrete problems. These Lagrange multipliers are unique and the discrete ones converge to the continuous ones. In order to derive the order of these convergences, the sufficient second order optimality conditions for the control problem are required. We prove that any local solution of the continuous control problem which is qualified and satisfies the sufficient second order optimality conditions can be uniformly approximated by discrete controls which are qualified local solutions of the discrete problems. Finally we obtain the order of these approximations.

First and second order optimality conditions play a crucial role in the numerical analysis of the control problems. Meanwhile the first order optimality conditions are known from long time ago, the second order conditions for optimal control problems governed by partial differential equations is a topic still under study,

\footnotetext{
Keywords and phrases: Distributed control, state constraints, semilinear elliptic equation, numerical approximation, finite element method, error estimates.

* This research was partially supported by Dirección General de Ense nanza Superior e Investigación Científica (Spain).

1 Dpt. Matemática Aplicada y Ciencias de la Computación, E.T.S.I.I y T., Universidad de Cantabria, Av. Los Castros s/n, 39005 Santander, Spain; e-mail: eduardo.casas@unican.es
} 
with some recent advances, but with a lot of work to be done yet. For this question the reader is referred to $[4,5,7-11,16,21]$.

There are no many papers devoted to the study of error estimates for the numerical discretization of control problems governed by partial differential equations. Let us mention two early papers devoted to linear-quadratic control problems by Falk [14] and Geveci [15]. A significant change when studying control problems with a nonlinear equation or a non quadratic functional is the necessity of using the sufficient second order optimality conditions to derive these error estimates. Recently Arada et al. [1] followed this procedure to get the error estimates for the same problem studied in this paper except by the fact that there were no state constraints. They derived the same $L^{\infty}$ error estimates than we obtain here. However in some cases they could take advantage of the absence of these constraints to deduce stronger $L^{2}$ error estimates than in this paper. This paper continues the research started in [1] by adding many finitely state constraints to the control problem. It is well known that this introduces a big difficulty in the approximation of the control problem and much extra work is necessary to deal with the state constraints. An essential assumption in this study is the qualification hypothesis (3.1) first used by Casas and Tröltzsch [8].

With respect to the optimality of the error estimates for the control, we can say that they seem to be optimal in the case of two dimensional domains, or in dimension three if the triangulation is of nonnegative type; see the final comments of the paper. To achieve these good estimates we have extended an idea of Malanowski et al. [18], also used in [1]. This idea leads to the definition of a variational inequality (6.27) close enough to that satisfied by the optimal control which appears in the first order optimality conditions. This variational inequality is compared with the one satisfied by the discrete optimal controls also deduced from the first order optimality conditions. See for instance [2] for a different method overestimating the error. In this paper the definition of the variational inequality (6.27) has required some new ideas and some extra work because of the presence of the state constraints.

The plan of the paper is as follows. In Section 2 the control problem is defined and the assumptions are listed. Also we summarize the differentiability results of the functionals involved in the problem. In Section 3 the first and second order optimality conditions are given without proofs. Some references are provided to check the proofs. The finite dimensional approximating problem is formulated in Section 4 . In this section we prove that qualified controls for the continuous problem can be approximated conveniently for feasible discrete controls. The existence of solutions for the final dimensional control problems is proved, whose main difficulty lies in proving that the set of feasible controls is non empty. First and second order optimality conditions for the discrete problems are stated in Section 5. Finally Section 6 is devoted to the study of the convergence of the discretization. The main results of the paper are presented in Section 4 and Section 6, in particular Theorem 6.8 is the main goal of this work.

\section{The CONTROL PROBlem}

Let $\Omega$ be an open convex set in $\mathbb{R}^{n}(n=2$ or 3$), \Gamma$ its boundary of class $C^{1,1}$ and $A$ an elliptic operator of the form

$$
A y=-\sum_{i, j=1}^{N} \partial_{x_{j}}\left[a_{i j} \partial_{x_{i}} y\right]+a_{0} y
$$

where the coefficients $a_{i j} \in C^{0,1}(\bar{\Omega})$ satisfy

$$
\lambda_{A}\|\xi\|^{2} \leq \sum_{i, j=1}^{n} a_{i j}(x) \xi_{i} \xi_{j} \quad \forall \xi \in \mathbb{R}^{n} \text { and } \forall x \in \Omega
$$

for some $\lambda_{A}>0$, and $a_{0} \in L^{\infty}(\Omega)$, with $a_{0}(x) \geq 0$. Let $f: \Omega \times \mathbb{R}^{2} \rightarrow \mathbb{R}$ and $L: \Omega \times \mathbb{R}^{2} \longrightarrow \mathbb{R}$ be Carathèodory functions. Given nonnegative integers $n_{e}$ and $n_{i}$, for every $1 \leq j \leq n_{e}+n_{i}$ we consider a function 
$F_{j}: C(\bar{\Omega}) \longrightarrow \mathbb{R}$. The control problem is formulated as follows

$$
(\mathrm{P})\left\{\begin{array}{l}
\min J(u)=\int_{\Omega} L\left(x, y_{u}(x), u(x)\right) \mathrm{d} x \\
\text { subject to }\left(y_{u}, u\right) \in\left(C(\bar{\Omega}) \cap H^{1}(\Omega)\right) \times L^{\infty}(\Omega) \\
\alpha \leq u(x) \leq \beta \quad \text { a.e. } \quad x \in \Omega \\
F_{j}\left(y_{u}\right)=0, \quad 1 \leq j \leq n_{e} \\
F_{j}\left(y_{u}\right) \leq 0, \quad n_{e}+1 \leq j \leq n_{e}+n_{i}
\end{array}\right.
$$

where $-\infty<\alpha<\beta<+\infty$ and $y_{u}$ is the solution of the state equation

$$
\left\{\begin{array}{l}
A y_{u}+f\left(\cdot, y_{u}\right)=u \text { in } \Omega \\
y_{u}=0 \text { on } \Gamma
\end{array}\right.
$$

Let us state the assumptions on the functionals $F_{j}, L$ and $f$.

(A1) $f$ is of class $C^{2}$ with respect to the second variable,

$$
f(\cdot, 0) \in L^{\infty}(\Omega), \quad \frac{\partial f}{\partial y}(x, y) \geq 0
$$

and for all $M>0$ there exists a constant $C_{f, M}>0$ such that

$$
\begin{aligned}
\left|\frac{\partial f}{\partial y}(x, y)\right|+\left|\frac{\partial^{2} f}{\partial y^{2}}(x, y)\right| & \leq C_{f, M} \text { for a.e. } x \in \Omega \text { and }|y| \leq M \\
\left|\frac{\partial^{2} f}{\partial y^{2}}\left(x, y_{2}\right)-\frac{\partial^{2} f}{\partial y^{2}}\left(x, y_{1}\right)\right| & <C_{f, M}\left|y_{2}-y_{1}\right| \text { for }\left|y_{1}\right|,\left|y_{2}\right| \leq M \text { and } x \in \Omega .
\end{aligned}
$$

(A2) $L: \Omega \times \mathbb{R} \times \mathbb{R} \longrightarrow \mathbb{R}$ is of class $C^{2}$ with respect to the second and third variables, $L(\cdot, 0,0) \in L^{1}(\Omega)$, and for all $M>0$ there exist a constant $C_{L, M}>0$ and a function $\psi_{M} \in L^{p}(\Omega)(p>n)$ such that

$$
\begin{gathered}
\left|\frac{\partial L}{\partial y}(x, y, u)\right| \leq \psi_{M}(x), \quad\left\|D_{(y, u)}^{2} L(x, y, u)\right\| \leq C_{L, M}, \\
\left|\frac{\partial L}{\partial u}\left(x_{2}, y, u\right)-\frac{\partial L}{\partial u}\left(x_{1}, y, u\right)\right| \leq C_{L, M}\left|x_{2}-x_{1}\right|, \\
\left\|D_{(y, u)}^{2} L\left(x, y_{2}, u_{2}\right)-D_{(y, u)}^{2} L\left(x, y_{1}, u_{1}\right)\right\| \leq C_{L, M}\left(\left|y_{2}-y_{1}\right|+\left|u_{2}-u_{1}\right|\right),
\end{gathered}
$$

for a.e. $x, x_{i} \in \Omega$ and $|y|,\left|y_{i}\right|,|u|,\left|u_{i}\right| \leq M, i=1,2$, where $D_{(y, u)}^{2} L$ denotes the second derivative of $L$ with respect to $(y, u)$. Moreover we assume that there exists $\lambda_{L}>0$ such that

$$
\frac{\partial^{2} L}{\partial u^{2}}(x, y, u) \geq \lambda_{L}, \quad \text { a.e. } x \in \Omega \text { and }(y, u) \in \mathbb{R}^{2} .
$$

(A3) For every $1 \leq j \leq n_{e}+n_{i}, F_{j}$ is of class $C^{2}$ in $C(\bar{\Omega}) ; F_{j}^{\prime}(y) \in L^{p}(\Omega)$ for every $y \in C(\bar{\Omega})$, for $p>n$ fixed; and for every $M>0$ there exists $C_{j, M}>0$ such that for every $1 \leq j \leq n_{e}+n_{i}$ and $\left\|y_{i}\right\|_{C(\bar{\Omega})} \leq M(i=1,2)$

$$
\left\|F_{j}^{\prime}\left(y_{2}\right)-F_{j}^{\prime}\left(y_{1}\right)\right\|_{L^{p}(\Omega)}+\left\|F_{j}^{\prime \prime}\left(y_{2}\right)-F_{j}^{\prime \prime}\left(y_{1}\right)\right\| \leq C_{j, M}\left\|y_{2}-y_{1}\right\|_{C(\bar{\Omega})}
$$


Typical state constraints defined by the functions $F_{j}$ are the integral constraints

$$
F_{j}(y)=\int_{\Omega} g_{j}(x, y(x)) \mathrm{d} x .
$$

Under the previous assumptions it is easy to prove the existence of a solution of Problem $(\mathrm{P})$ assuming that the set of feasible controls is not empty. In the proof it is essential the convexity of $L$ with respect to the control. In (A2) we have assumed that $L$ is strictly convex with respect to $u$, which will be useful to prove the strong convergence of the discretizations. Therefore this strong convexity is not a too restrictive assumption if we want to have a well posed problem in the sense that it has at least one solution. However there is a situation which is interesting in practice and it is not included in our formulation. This is the case of a function $L$ depending only on the variables $(x, y)$, but not on $u$. The optimal control problem is typically bang-bang in this situation. It is an open problem for us the derivation of the error estimates in the bang-bang case.

Among the functionals included in our problem, we can consider those of the type $L(x, y, u)=g(x, y)+h(u)$, with $h^{\prime \prime}(u) \geq \lambda_{L}$. For instance, the classical example $L(x, y, u)=\left(y-y_{d}(x)\right)^{2}+N u^{2}$, with $N>0$ is of this type.

We finish this section by recalling some results about the differentiability of the functionals involve in the control problem. For the detailed proofs the reader is referred to Casas and Mateos [5].

Theorem 2.1. Suppose (A1) holds. Then for every $u \in L^{\infty}(\Omega)$, the state equation (2.1) has a unique solution $y_{u}$ in the space $W^{2, p}(\Omega)$ and the mapping $G: L^{\infty}(\Omega) \longrightarrow W^{2, p}(\Omega)$, defined by $G(u)=y_{u}$ is of class $C^{2}$. Moreover for all $v, u \in L^{\infty}(\Omega), z_{v}=G^{\prime}(u) v$ is defined as the solution of

$$
\left\{\begin{array}{l}
A z_{v}+\frac{\partial f}{\partial y}\left(x, y_{u}\right) z_{v}=v \text { in } \Omega \\
z_{v}=0 \text { on } \Gamma .
\end{array}\right.
$$

Finally, for every $v_{1}, v_{2} \in L^{\infty}(\Omega), z_{v_{1} v_{2}}=G^{\prime \prime}(u) v_{1} v_{2}$ is the solution of

$$
\left\{\begin{aligned}
A z_{v_{1} v_{2}}+\frac{\partial f}{\partial y}\left(x, y_{u}\right) z_{v_{1} v_{2}}+\frac{\partial^{2} f}{\partial y^{2}}\left(x, y_{u}\right) z_{v_{1}} z_{v_{2}} & =0 \text { in } \Omega \\
z_{v_{1} v_{2}} & =0 \text { on } \Gamma
\end{aligned}\right.
$$

where $z_{v_{i}}=G^{\prime}(u) v_{i}, i=1,2$.

The $W^{2, p}(\Omega)$ regularity is an immediate consequence of our assumptions; see Grisvard [17]. The rest can be obtained by using the implicit function theorem.

Theorem 2.2. Let us suppose that (A1) and (A2) hold. Then the functional $J: L^{\infty}(\Omega) \rightarrow \mathbb{R}$ is of class $C^{2}$. Moreover, for every $u, v, v_{1}, v_{2} \in L^{\infty}(\Omega)$

$$
J^{\prime}(u) v=\int_{\Omega}\left(\frac{\partial L}{\partial u}\left(x, y_{u}, u\right)+\varphi_{0 u}\right) v \mathrm{~d} x
$$

and

$$
\begin{aligned}
J^{\prime \prime}(u) v_{1} v_{2}= & \int_{\Omega}\left[\frac{\partial^{2} L}{\partial y^{2}}\left(x, y_{u}, u\right) z_{v_{1}} z_{v_{2}}+\frac{\partial^{2} L}{\partial y \partial u}\left(x, y_{u}, u\right)\left(z_{v_{1}} v_{2}+z_{v_{2}} v_{1}\right)\right. \\
& \left.+\frac{\partial^{2} L}{\partial u^{2}}\left(x, y_{u}, u\right) v_{1} v_{2}-\varphi_{0 u} \frac{\partial^{2} f}{\partial y^{2}}\left(x, y_{u}\right) z_{v_{1}} z_{v_{2}}\right] \mathrm{d} x
\end{aligned}
$$


where $y_{u}=G(u), \varphi_{0 u} \in W^{2, p}(\Omega)$ is the unique solution of the problem

$$
\left\{\begin{array}{l}
A^{*} \varphi+\frac{\partial f}{\partial y}\left(x, y_{u}\right) \varphi=\frac{\partial L}{\partial y}\left(x, y_{u}, u\right) \text { in } \Omega \\
\varphi=0 \text { on } \Gamma
\end{array}\right.
$$

where $A^{*}$ is the adjoint operator of $A$ and $z_{v_{i}}=G^{\prime}(u) v_{i}, i=1,2$.

This theorem follows from Theorem 2.1 and the chain rule.

Theorem 2.3. Let us suppose that $(\mathbf{A 1})$ and (A3) hold. Then for each $j$, the functional $G_{j}=F_{j} \circ G$ : $L^{\infty}(\Omega) \rightarrow \mathbb{R}$ is of class $C^{2}$. Moreover, for every $u, v, v_{1}, v_{2} \in L^{\infty}(\Omega)$

$$
G_{j}^{\prime}(u) v=\int_{\Omega} \varphi_{j u} v \mathrm{~d} x
$$

and

$$
G_{j}^{\prime \prime}(u) v_{1} v_{2}=F_{j}^{\prime \prime}\left(y_{u}\right) z_{v_{1}} z_{v_{2}}-\int_{\Omega} \varphi_{j u} \frac{\partial^{2} f}{\partial y^{2}}\left(x, y_{u}\right) z_{v_{1}} z_{v_{2}} \mathrm{~d} x
$$

where $y_{u}=G(u), \varphi_{j u} \in W^{2, p}(\Omega)$ is the unique solution of the problem

$$
\left\{\begin{array}{l}
A^{*} \varphi+\frac{\partial f}{\partial y}\left(x, y_{u}\right) \varphi=F_{j}^{\prime}\left(y_{u}\right) \text { in } \Omega \\
\varphi=0 \text { on } \Gamma
\end{array}\right.
$$

and $z_{v_{i}}=G^{\prime}(u) v_{i}, i=1,2$.

\section{FIRST AND SECOND ORDER OPTIMALITY CONDITIONS}

We start this sections by reformulating problem $(\mathrm{P})$ with the help of the functionals $G_{j}=F_{j} \circ G$ introduced in Theorems 2.1 and 2.3.

$$
(\mathrm{P})\left\{\begin{array}{l}
\text { Minimize } J(u) \\
\alpha \leq u(x) \leq \beta \text { a.e. } x \in \Omega, \\
G_{j}(u)=0,1 \leq j \leq n_{e} \\
G_{j}(u) \leq 0, n_{e}+1 \leq j \leq n_{e}+n_{i} .
\end{array}\right.
$$

In order to state the optimality conditions for a local solution of $(\mathrm{P})$ we introduce some notation. Fixed a feasible control $\bar{u}$ and given $\varepsilon>0$, we denote the set of $\varepsilon$-inactive constraints by

$$
\Omega_{\varepsilon}=\{x \in \Omega: \alpha+\varepsilon \leq \bar{u}(x) \leq \beta-\varepsilon\}
$$

We say that a feasible control $\bar{u}$ is regular if the following assumption is fulfilled

$$
\left\{\begin{array}{l}
\exists \varepsilon_{\bar{u}}>0 \text { and }\left\{\bar{w}_{j}\right\}_{j \in I_{0}} \subset L^{\infty}(\Omega), \text { with supp } \bar{w}_{j} \subset \Omega_{\varepsilon_{\bar{u}}}, \text { such that } \\
G_{i}^{\prime}(\bar{u}) \bar{w}_{j}=\delta_{i j}, \quad i, j \in I_{0},
\end{array}\right.
$$

where

$$
I_{0}=\left\{j \leq n_{e}+n_{i} \mid G_{j}(\bar{u})=0\right\}
$$


$I_{0}$ is the set of indices corresponding to active constraints. Following lemma proves that functions $\left\{\bar{w}_{j}\right\}_{j \in I_{0}}$ can be chosen of class $C^{\infty}$.

Lemma 3.1. Let us assume that $\bar{u}$ is continuous in $\bar{\Omega}$ and satisfies (3.1), then for any $\varepsilon<\varepsilon_{\bar{u}}$ there exist some functions $\left\{\tilde{w}_{j}\right\}_{j \in I_{0}} \subset C^{\infty}(\bar{\Omega})$ with support in $\Omega_{\varepsilon}$ such that $G_{i}^{\prime}(\bar{u}) \tilde{w}_{j}=\delta_{i j}$.

Proof. Let $\varepsilon<\varepsilon_{\bar{u}}$, then by extending the functions $\left\{\bar{w}_{j}\right\}_{j \in I_{0}}$ given in (3.1) by zero to $\mathbb{R}^{n}$ and making the convolution with a regularizing sequence we get functions $\left\{\bar{w}_{j k}\right\}_{k=1}^{\infty} \subset C^{\infty}\left(\mathbb{R}^{n}\right)$, for every $j \in I_{0}$, converging to $\bar{w}_{j}$ in $L^{p}(\Omega)$. Moreover, since $\bar{u}$ is continuous we have that $\Omega_{\varepsilon_{\bar{u}}} \subset \Omega_{\varepsilon}$ and then for $k$ large enough $\operatorname{supp}\left(\bar{w}_{j k}\right) \subset \Omega_{\varepsilon}$.

Since $\bar{w}_{j k} \rightarrow \bar{w}_{j}$ in $L^{p}(\Omega)$, we deduce from $(2.7)$ that $G_{i}^{\prime}(\bar{u}) \bar{w}_{j k} \rightarrow G_{i}^{\prime}(\bar{u}) \bar{w}_{j}$ for every $i, j \in I_{0}$. Denoting by $m$ the number of elements of $I_{0}$ and using this convergence, we can deduce the existence of $k_{0}$ such that

$$
\left|\delta_{i j}-G_{i}^{\prime}(\bar{u}) \bar{w}_{j k}\right|<\frac{1}{m} \quad \forall k \geq k_{0} \text { and } i, j \in I_{0} .
$$

From these inequalities we deduce that the vectors $\left\{\left(G_{i}^{\prime}(\bar{u}) \bar{w}_{j k}\right)_{i \in I_{0}}\right\}_{j \in I_{0}} \subset \mathbb{R}^{m}$ are linearly independent. Indeed let us take scalars $\left\{c_{j}\right\}_{j \in I_{0}}$ such that

$$
\sum_{j \in I_{0}} c_{j}\left(G_{i}^{\prime}(\bar{u}) \bar{w}_{j k}\right)_{i \in I_{0}}=0
$$

Let $\left|c_{l}\right|=\max \left\{\left|c_{j}\right|: j \in I_{0}\right\}$. Then

$$
\sum_{j \in I_{0}, j \neq l} c_{j}\left(G_{i}^{\prime}(\bar{u}) \bar{w}_{j k}\right)_{i \in I_{0}}=-c_{l}\left(G_{i}^{\prime}(\bar{u}) \bar{w}_{l k}\right)_{i \in I_{0}}
$$

Assuming $c_{l} \neq 0$, from this identity and (3.2) it follows

$$
\left(1-\frac{1}{m}\right)\left|c_{l}\right|<\left|c_{l} G_{l}^{\prime}(\bar{u}) \bar{w}_{l k}\right| \leq \sum_{j \in I_{0}, j \neq l}\left|c_{j}\right|\left|G_{l}^{\prime}(\bar{u}) \bar{w}_{j k}\right| \leq\left|c_{l}\right| \frac{m-1}{m},
$$

which is a contradiction, therefore $c_{l}=0$. Consequently we have that the linear mapping $S_{k}: \mathbb{R}^{m} \longrightarrow \mathbb{R}^{m}$ defined by

$$
S_{k}(c)=\left(G_{i}^{\prime}(\bar{u})\left[\sum_{j \in I_{0}} c_{j} \bar{w}_{j k}\right]\right)_{i \in I_{0}}
$$

is an isomorphism. Therefore if we denote by $\left\{e_{j}\right\}_{j \in I_{0}}$ the canonical base of $\mathbb{R}^{m}$, then we deduce the existence of vectors $c_{k}^{i}=\left(c_{j k}^{i}\right)_{j \in I_{0}}$ such that $S_{k}\left(c_{k}^{i}\right)=e_{i}$. Now setting

$$
\tilde{w}_{i k}=\sum_{j \in I_{0}} c_{j k}^{i} \bar{w}_{j k}
$$

we have that $G_{i}^{\prime}(\bar{u}) \tilde{w}_{j k}=\delta_{i j}$ and $\left\{\tilde{w}_{j k}\right\}_{j \in I_{0}}$ satisfies the requirements of the lemma.

Associated with problem (P) we consider the usual Lagrangian function $\mathcal{L}: L^{\infty}(\Omega) \times \mathbb{R}^{n_{e}+n_{i}} \longrightarrow \mathbb{R}$ given by

$$
\mathcal{L}(u, \lambda)=J(u)+\sum_{j=1}^{n_{e}+n_{i}} \lambda_{j} G_{j}(u) .
$$


Obviously (3.1) is equivalent to the linear independence of the derivatives $\left\{G_{j}^{\prime}(\bar{u})\right\}_{j \in I_{0}}$ in $L^{1}\left(\Omega_{\varepsilon_{\bar{u}}}\right)$. Under this assumption we can derive the first order necessary conditions for optimality in a qualified form. For the proof the reader is referred to Bonnans and Casas [3] or Clarke [13]; see also Mateos [19].

Theorem 3.2. Let us assume that $\bar{u}$ is a local solution of $(\mathrm{P})$ and (3.1) holds. Then there exist real numbers $\left\{\bar{\lambda}_{j}\right\}_{j=1}^{n_{e}+n_{i}}$ such that

$$
\begin{gathered}
\bar{\lambda}_{j} \geq 0 \quad \text { and } \bar{\lambda}_{j} G_{j}(\bar{u})=0, \quad \text { if } n_{e}+1 \leq j \leq n_{e}+n_{i} \\
\frac{\partial \mathcal{L}}{\partial u}(\bar{u}, \bar{\lambda})(u-\bar{u}) \geq 0 \quad \text { for all } \alpha \leq u \leq \beta
\end{gathered}
$$

Denoting by $\bar{\varphi}_{0}$ and $\bar{\varphi}_{j}$ the solutions of (2.6) and (2.1) corresponding to $\bar{u}$ and setting

$$
\bar{\varphi}=\bar{\varphi}_{0}+\sum_{j=1}^{n_{e}+n_{i}} \bar{\lambda}_{j} \bar{\varphi}_{j}
$$

we deduce from Theorems 2.2 and 2.3 and the definition of $\mathcal{L}$ that

$$
\begin{aligned}
\frac{\partial \mathcal{L}}{\partial u}(\bar{u}, \bar{\lambda}) v & =\int_{\Omega}\left(\frac{\partial L}{\partial u}(x, \bar{y}, \bar{u})+\bar{\varphi}_{0}\right) v \mathrm{~d} x+\sum_{j=1}^{n_{e}+n_{i}} \bar{\lambda}_{j} \int_{\Omega} \bar{\varphi}_{j} v \mathrm{~d} x \\
& =\int_{\Omega}\left(\frac{\partial L}{\partial u}(x, \bar{y}, \bar{u})+\bar{\varphi}\right) v \mathrm{~d} x=\int_{\Omega} \mathrm{d}(x) v(x) \quad \forall v \in L^{\infty}(\Omega),
\end{aligned}
$$

where $\bar{y}=G(\bar{u})=y_{\bar{u}}$ and

$$
d(x)=\frac{\partial L}{\partial u}(x, \bar{y}(x), \bar{u}(x))+\bar{\varphi}(x)
$$

From (3.4) we deduce that

$$
d(x)=\left\{\begin{array}{cl}
0 & \text { for a.e. } x \in \Omega \text { where } \alpha<\bar{u}(x)<\beta \\
\geq 0 & \text { for a.e. } x \in \Omega \text { where } \bar{u}(x)=\alpha \\
\leq 0 & \text { for a.e. } x \in \Omega \text { where } \bar{u}(x)=\beta
\end{array}\right.
$$

Remark 3.3. From $(3.4,3.7)$ and assumption (3.1) we get

$$
\int_{\Omega}\left(\frac{\partial L}{\partial u}(x, \bar{y}(x), \bar{u}(x))+\bar{\varphi}_{0}(x)\right) \bar{w}_{j}(x) \mathrm{d} x+\bar{\lambda}_{j}=\frac{\partial \mathcal{L}}{\partial u}(\bar{u}, \bar{\lambda}) \bar{w}_{j}=0,
$$

which implies the uniqueness of the Lagrange multipliers provided in Theorem 3.2.

Associated with $d$ we set

$$
\Omega^{0}=\{x \in \Omega:|d(x)|>0\} .
$$

Given $\left\{\bar{\lambda}_{j}\right\}_{j=1}^{n_{e}+n_{i}}$ by Theorem 3.2, we define the cone of critical directions

$$
C_{\bar{u}}^{0}=\left\{v \in L^{2}(\Omega) \text { satisfying (3.11) and } v(x)=0 \text { for a.e. } x \in \Omega^{0}\right\}
$$


with

$$
\left\{\begin{array}{l}
G_{j}^{\prime}(\bar{u}) v=0 \text { if }\left(j \leq n_{e}\right) \text { or }\left(j>n_{e}, G_{j}(\bar{u})=0 \text { and } \bar{\lambda}_{j}>0\right) \\
G_{j}^{\prime}(\bar{u}) v \leq 0 \text { if }\left(j>n_{e}, G_{j}(\bar{u})=0 \text { and } \bar{\lambda}_{j}=0\right) \\
v(x)= \begin{cases}\geq 0 & \text { if } \bar{u}(x)=\alpha \\
\leq 0 & \text { if } \bar{u}(x)=\beta\end{cases}
\end{array}\right.
$$

Now we are ready to state the second order necessary optimality conditions.

Theorem 3.4. Let us assume that $\bar{u}$ is a local solution of $(\mathrm{P})$, equation (3.1) holds and $\left\{\bar{\lambda}_{j}\right\}_{j=1}^{m}$ are the Lagrange multipliers satisfying (3.3) and (3.4). Then the following inequality is satisfied

$$
\frac{\partial^{2} \mathcal{L}}{\partial u^{2}}(\bar{u}, \bar{\lambda}) v^{2} \geq 0 \quad \forall v \in C_{\bar{u}}^{0}
$$

For the proof see Casas and Tröltzsch [9] and Casas and Mateos ([5], Th. 3.3 and Prop. 3.6). The sufficient optimality conditions can be formulated as follows:

Theorem 3.5. Let $\bar{u}$ be an admissible control for problem (P) satisfying the regularity assumption (3.1) and (3.3-3.4) for some $\bar{\lambda}_{j}, j=1, \ldots, n_{i}+n_{e}$. Let us suppose also that

$$
\frac{\partial^{2} \mathcal{L}}{\partial u^{2}}(\bar{u}, \bar{\lambda}) v^{2}>0 \text { for all } v \in C_{\bar{u}}^{0} \backslash\{0\}
$$

Then there exist $\bar{\varepsilon}>0$ and $\bar{\mu}>0$ such that $J(\bar{u})+\bar{\mu}\|u-\bar{u}\|_{L^{2}(\Omega)}^{2} \leq J(u)$ for all admissible control $u$ with $\|u-\bar{u}\|_{L^{\infty}(\Omega)} \leq \bar{\varepsilon}$.

Taking into account that the Hamiltonian of problem $(\mathrm{P})$ is

$$
H(x, y, u, \varphi)=L(x, y, u)+\varphi[u-f(x, y)]
$$

and according to the Assumption (A2)

$$
\frac{\partial^{2} H}{\partial u^{2}}(x, \bar{y}(x), \bar{u}(x), \bar{\varphi}(x))=\frac{\partial^{2} L}{\partial u^{2}}(x, \bar{y}(x), \bar{u}(x)) \geq \lambda_{L}>0 \quad \text { a.e. } \quad x \in \Omega,
$$

then Theorem 3.5 is an immediate consequence of [5] (Th. 4.3).

The gap between the necessary and sufficient optimality conditions for problem $(\mathrm{P})$ is minimal. In fact, strictly speaking, there is no gap because whenever $\bar{u}$ is a strict local solution of $(\mathrm{P})$ (in the sense of Th. 3.5), then (3.13) holds. To deduce this it is enough to notice that $\bar{u}$ is a local solution of the problem

$$
\left(\mathrm{P}_{\mu}\right)\left\{\begin{array}{l}
\text { Minimize } J_{\mu}(u)=J(u)-\bar{\mu}\|u-\bar{u}\|_{L^{2}(\Omega)}^{2}, \\
\alpha \leq u(x) \leq \beta \text { a.e. } x \in \Omega, \\
G_{j}(u)=0,1 \leq j \leq n_{e} \\
G_{j}(u) \leq 0, n_{e}+1 \leq j \leq n_{e}+n_{i},
\end{array}\right.
$$

and to apply Theorem 3.4 to this problem and to use that

$$
0 \leq \frac{\partial^{2} \mathcal{L}_{\mu}}{\partial u^{2}}(\bar{u}, \bar{\lambda}) v^{2}=\frac{\partial^{2} \mathcal{L}}{\partial u^{2}}(\bar{u}, \bar{\lambda}) v^{2}-2 \bar{\mu}\|v\|_{L^{2}(\Omega)}^{2} \quad \forall v \in C_{\bar{u}}^{0} .
$$


In particular we have obtained that condition (3.13) implies that

$$
\frac{\partial^{2} \mathcal{L}}{\partial u^{2}}(\bar{u}, \bar{\lambda}) v^{2} \geq 2 \bar{\mu}\|v\|_{L^{2}(\Omega)}^{2} \quad \forall v \in C_{\bar{u}}^{0} .
$$

By using Theorem 4.4 of [5], we have even more:

Theorem 3.6. Let $\bar{u}$ be an admissible control for problem $(\mathrm{P})$ that satisfies $(\mathbf{A} \mathbf{1}-\mathbf{A} \mathbf{3})$, the regularity assumptions (3.1) and (3.3, 3.4). Then (3.13) is equivalent to the existence of $\bar{\mu}>0$ and $\bar{\tau}>0$ such that

$$
\frac{\partial^{2} \mathcal{L}}{\partial u^{2}}(\bar{u}, \bar{\lambda}) v^{2} \geq \bar{\mu}\|v\|_{L^{2}(\Omega)}^{2} \text { for all } v \in C_{\bar{u}}^{\bar{\tau}}
$$

where

$$
C_{\bar{u}}^{\bar{\tau}}=\left\{v \in L^{2}(\Omega) \text { satisfying (3.11) and } v(x)=0 \text { a.e. } x \in \Omega^{\bar{\tau}}\right\},
$$

and

$$
\Omega^{\bar{\tau}}=\{x \in \Omega:|d(x)|>\bar{\tau}\}
$$

We finish this section by providing a characterization of the optimal control $\bar{u}$.

Theorem 3.7. Suppose that $\bar{u}$ is a local solution of $(\mathrm{P})$ and assumptions (A1-A3) and (3.1) are satisfied. Then, for all $x \in \bar{\Omega}$, the equation

$$
\varphi_{\bar{u}}(x)+\frac{\partial L}{\partial u}\left(x, y_{\bar{u}}(x), t\right)=0
$$

has a unique solution $\bar{t}=\bar{s}(x)$. The mapping $\bar{s}: \bar{\Omega} \longrightarrow \mathbb{R}$ is Lipschitz. Moreover $\bar{u}$ and $\bar{s}$ are related by the formula

$$
\bar{u}(x)=\operatorname{Proj}_{[\alpha, \beta]}(\bar{s}(x))=\max (\alpha, \min (\beta, \bar{s}(x))),
$$

and $\bar{u}$ also belongs to $C^{0,1}(\bar{\Omega})$.

The proof of the existence and uniqueness of a solution of $(3.15)$ is a consequence of $\left(\partial^{2} L / \partial u^{2}\right)(x, y, u)$ $\geq \lambda_{L}>0$. The Lipschitz regularity of $\bar{s}$ follows from the Lipschitz properties of $L$ (Assumption (A2)) and the fact that $y_{\bar{u}}, \varphi_{\bar{u}} \in W^{2, p}(\Omega) \subset C^{0,1}(\bar{\Omega})$. For the details see Arada et al. [1].

\section{Finite-element approximation of $(\mathrm{P})$}

Here we define a finite-element based approximation of the optimal control problem $(P)$. To this aim, we consider a family of triangulations $\left\{\mathcal{T}_{h}\right\}_{h>0}$ of $\bar{\Omega}$. This triangulation is supposed to be regular in the usual sense that we state exactly here. With each element $T \in \mathcal{T}_{h}$, we associate two parameters $\rho(T)$ and $\sigma(T)$, where $\rho(T)$ denotes the diameter of the set $T$ and $\sigma(T)$ is the diameter of the largest ball contained in $T$. Define the size of the mesh by $h=\max _{T \in \mathcal{T}_{h}} \rho(T)$. We suppose that the following regularity assumptions are satisfied.

(i) There exist two positive constants $\rho$ and $\sigma$ such that

$$
\frac{\rho(T)}{\sigma(T)} \leq \sigma, \quad \frac{h}{\rho(T)} \leq \rho
$$

hold for all $T \in \mathcal{T}_{h}$ and all $h>0$. 
(ii) Let us take $\bar{\Omega}_{h}=\cup_{T \in \mathcal{T}_{h}} T$, and let $\Omega_{h}$ and $\Gamma_{h}$ denote its interior and its boundary, respectively. We assume that $\bar{\Omega}_{h}$ is convex and that the vertices of $\mathcal{T}_{h}$ placed on the boundary of $\Gamma_{h}$ are points of $\Gamma$. From [20] (estimate (5.2.19)) we know

$$
\left|\Omega \backslash \Omega_{h}\right| \leq C h^{2} .
$$

Now, to every boundary triangle $T$ of $\mathcal{T}_{h}$, we associate another triangle $\hat{T} \subset \bar{\Omega}$ with curved boundary as follows: the edge between the two boundary nodes of $T$ is substituted by the part of $\Gamma$ connecting these nodes and forming a triangle with the remaining interior sides of $T$. We denote by $\widehat{\mathcal{T}}_{h}$ the family of these curved boundary triangles along with the interior triangles to $\Omega$ of $\mathcal{T}_{h}$, so that $\bar{\Omega}=\cup_{\hat{T} \in \widehat{\mathcal{T}}_{h}} \hat{T}$. Let us set

$$
\begin{aligned}
U_{h} & =\left\{u \in L^{\infty}(\Omega) \mid u_{\mid \hat{T}} \text { is constant on all } \hat{T} \in \widehat{\mathcal{T}}_{h}\right\}, \\
Y_{h} & =\left\{y_{h} \in C(\bar{\Omega}) \mid y_{h \mid T} \in \mathcal{P}_{1}, \text { for all } T \in \mathcal{T}_{h}, \text { and } y_{h}=0 \text { on } \bar{\Omega} \backslash \Omega_{h}\right\},
\end{aligned}
$$

where $\mathcal{P}_{1}$ is the space of polynomials of degree less or equal than 1 . For each $u \in L^{\infty}(\Omega)$, we denote by $y_{h}(u)$ the unique element of $Y_{h}$ that satisfies

$$
a\left(y_{h}(u), z_{h}\right)+\int_{\Omega} f\left(x, y_{h}(u)\right) z_{h}(x) \mathrm{d} x=\int_{\Omega} u(x) z_{h}(x) \mathrm{d} x \quad \forall z_{h} \in Y_{h},
$$

where $a: Y_{h} \times Y_{h} \longrightarrow \mathbb{R}$ is the bilinear form defined by

$$
a\left(y_{h}, z_{h}\right)=\int_{\Omega}\left(\sum_{i, j=1}^{n} a_{i j}(x) \partial_{x_{i}} y_{h}(x) \partial_{x_{j}} z_{h}(x)+a_{0}(x) y_{h}(x) z_{h}(x)\right) \mathrm{d} x .
$$

In other words, $y_{h}(u)$ is the approximate state associated with $u$. Notice that $y_{h}=z_{h}=0$ on $\bar{\Omega} \backslash \bar{\Omega}_{h}$, hence the last integral is equivalent to integration on $\Omega_{h}$. The finite dimensional approximation of the optimal control problem is defined by

$$
\left(\mathrm{P}_{h}\right)\left\{\begin{array}{l}
\min J_{h}\left(u_{h}\right)=\int_{\Omega_{h}} L\left(x, y_{h}\left(u_{h}\right)(x), u_{h}(x)\right) \mathrm{d} x, \\
\text { subject to }\left(y_{h}\left(u_{h}\right), u_{h}\right) \in Y_{h} \times U_{h}, \\
\alpha \leq u_{h}(x) \leq \beta \quad \text { a.e. } \quad x \in \Omega_{h}, \\
F_{j}\left(y_{h}\left(u_{h}\right)=0, \quad 1 \leq j \leq n_{e}\right. \\
F_{j}\left(y_{h}\left(u_{h}\right)\right) \leq 0, \quad n_{e}+1 \leq j \leq n_{e}+n_{i} .
\end{array}\right.
$$

For every $h>0$ let us define $G_{h}: L^{\infty}(\Omega) \longrightarrow \mathbb{R}$ and $G_{h j}: L^{\infty}(\Omega) \longrightarrow Y_{h}\left(1 \leq j \leq n_{e}+n_{i}\right)$ by $G_{h}(u)=y_{h}(u)$ and $G_{h j}(u)=\left(F_{j} \circ G_{h}\right)(u)=F_{j}\left(y_{h}(u)\right)$. Then problem $\left(\mathrm{P}_{h}\right)$ can be written as follows

$$
\left(\mathrm{P}_{h}\right)\left\{\begin{array}{l}
\text { Minimize } J_{h}\left(u_{h}\right) \\
u_{h} \in U_{h}, \alpha \leq u_{h}(x) \leq \beta \text { a.e. } x \in \Omega_{h} \\
G_{h j}\left(u_{h}\right)=0,1 \leq j \leq n_{e} \\
G_{h j}\left(u_{h}\right) \leq 0, n_{e}+1 \leq j \leq n_{e}+n_{i}
\end{array}\right.
$$

We start the study of problem $\left(\mathrm{P}_{h}\right)$ by analyzing the differentiability of the functions involved in the control problem. Let us collect the differentiability results analogous to those of Section 2.

Theorem 4.1. Suppose (A1) holds. Then for every $u \in L^{\infty}(\Omega)$, the problem (4.2) has a unique solution $y_{h}(u) \in Y_{h}$ and the mapping $G_{h}: L^{\infty}(\Omega) \longrightarrow Y_{h}$, defined by $G_{h}(u)=y_{h}(u)$ is of class $C^{2}$ and for all 
$v, u \in L^{\infty}(\Omega), z_{h}(v)=G_{h}^{\prime}(u) v$ is the solution of

$$
a\left(z_{h}(v), q_{h}\right)+\int_{\Omega} \frac{\partial f}{\partial y}\left(x, y_{h}(u)\right) z_{h}(v) q_{h} \mathrm{~d} x=\int_{\Omega} v q_{h} \mathrm{~d} x \quad \forall q_{h} \in Y_{h} .
$$

Finally, for every $v_{1}, v_{2} \in L^{\infty}(\Omega), z_{h}\left(v_{1}, v_{2}\right)=G^{\prime \prime}(u) v_{1} v_{2}$ is the solution of

$$
a\left(z_{h}, q_{h}\right)+\int_{\Omega} \frac{\partial f}{\partial y}\left(x, y_{h}(u)\right) z_{h} q_{h} \mathrm{~d} x+\int_{\Omega} \frac{\partial^{2} f}{\partial y^{2}}\left(x, y_{h}(u)\right) z_{h}\left(v_{1}\right) z_{h}\left(v_{2}\right) q_{h} \mathrm{~d} x=0 \quad \forall q_{h} \in Y_{h},
$$

where $z_{h}\left(v_{i}\right)=G_{h}^{\prime}(u) v_{i}, i=1,2$.

Theorem 4.2. Let us suppose that (A1) and (A2) hold. Then the functional $J_{h}: L^{\infty}(\Omega) \rightarrow \mathbb{R}$ is of class $C^{2}$. Moreover, for every $u, v, v_{1}, v_{2} \in L^{\infty}(\Omega)$

$$
J_{h}^{\prime}(u) v=\int_{\Omega_{h}}\left(\frac{\partial L}{\partial u}\left(x, y_{h}(u), u\right)+\varphi_{h 0}(u)\right) v \mathrm{~d} x
$$

and

$$
\begin{aligned}
J_{h}^{\prime \prime}(u) v_{1} v_{2}= & \int_{\Omega_{h}}\left[\frac{\partial^{2} L}{\partial y^{2}}\left(x, y_{h}(u), u\right) z_{h}\left(v_{1}\right) z_{h}\left(v_{2}\right)+\frac{\partial^{2} L}{\partial y \partial u}\left(x, y_{h}(u), u\right)\left[z_{h}\left(v_{1}\right) v_{2}+z_{h}\left(v_{2}\right) v_{1}\right]\right. \\
& \left.+\frac{\partial^{2} L}{\partial u^{2}}\left(x, y_{h}(u), u\right) v_{1} v_{2}-\varphi_{h 0}(u) \frac{\partial^{2} f}{\partial y^{2}}\left(x, y_{h}(u)\right) z_{h}\left(v_{1}\right) z_{h}\left(v_{2}\right)\right] \mathrm{d} x
\end{aligned}
$$

where $y_{h}(u)=G_{h}(u), \varphi_{h 0}(u) \in Y_{h}$ is the unique solution of the problem

$$
a\left(q_{h}, \varphi_{h 0}(u)\right)+\int_{\Omega} \frac{\partial f}{\partial y}\left(x, y_{h}(u)\right) \varphi_{h 0}(u) q_{h} \mathrm{~d} x=\int_{\Omega} \frac{\partial L}{\partial y}\left(x, y_{h}(u), u\right) q_{h} \mathrm{~d} x \quad \forall q_{h} \in Y_{h},
$$

with $z_{h}\left(v_{i}\right)=G_{h}^{\prime}(u) v_{i}, i=1,2$.

Theorem 4.3. Let us suppose that (A1) and (A3) hold. Then for each $j$, the functional $G_{h j}=F_{j} \circ G_{h}$ : $L^{\infty}(\Omega) \rightarrow \mathbb{R}$ is of class $C^{2}$. Moreover, for every $u, v, v_{1}, v_{2} \in L^{\infty}(\Omega)$

$$
G_{h j}^{\prime}(u) v=\int_{\Omega} \varphi_{h j}(u) v \mathrm{~d} x
$$

and

$$
G_{h j}^{\prime \prime}(u) v_{1} v_{2}=F_{j}^{\prime \prime}\left(y_{h}(u)\right) z_{h}\left(v_{1}\right) z_{h}\left(v_{2}\right)-\int_{\Omega} \varphi_{h j}(u) \frac{\partial^{2} f}{\partial y^{2}}\left(x, y_{h}(u)\right) z_{h}\left(v_{1}\right) z_{h}\left(v_{2}\right) \mathrm{d} x
$$

where $y_{h}(u)=G_{h}(u), \varphi_{h j}(u) \in Y_{h}$ is the unique solution of the variational equation

$$
a\left(q_{h}, \varphi_{h j}(u)\right)+\int_{\Omega} \frac{\partial f}{\partial y}\left(x, y_{h}(u)\right) \varphi_{h j}(u) q_{h} \mathrm{~d} x=\int_{\Omega_{h}} F_{j}^{\prime}\left(y_{h}(u)\right) q_{h} \quad \forall q_{h} \in Y_{h}
$$

and $z_{h}\left(v_{i}\right)=G_{h}^{\prime}(u) v_{i}, i=1,2$.

Our next goal is to study the existence of a solution of $\left(\mathrm{P}_{h}\right)$. The difficulty consists in proving that the set of admissible discrete controls

$$
\begin{gathered}
U_{h a d}=\left\{u_{h} \in U_{h}: \alpha \leq u_{h}(x) \leq \beta \text { a.e. } x \in \Omega_{h}, G_{h j}\left(u_{h}\right)=0,1 \leq j \leq n_{e}\right. \\
\left.G_{h j}\left(u_{h}\right) \leq 0, n_{e}+1 \leq j \leq n_{e}+n_{i}\right\}
\end{gathered}
$$


is not empty. To deal with this question we will use the classical approximation operator $\Pi_{h}: L^{1}(\Omega) \longrightarrow U_{h}$ defined as follows: $u_{h}=\Pi_{h} u$ is the element of $U_{h}$ such that

$$
u_{h \mid T}=\frac{1}{|T|} \int_{T} u(x) \mathrm{d} x
$$

for every $T \in \mathcal{T}_{h}$. Due to the state constraints, we do not have, as usual, that the projections $\Pi_{h} u$ of feasible controls $u$ for $(\mathrm{P})$ are feasible controls for $\left(\mathrm{P}_{h}\right)$. The regularity assumption (3.1) plays an essential role in this approximation analysis. Another crucial point is the study of the convergence of the discretization of the state and adjoint state equations. Here we will use the following two results whose proofs can be found in [1] and [6].

Lemma 4.4. Let $\left(v, v_{h}\right) \in L^{\infty}(\Omega) \times U_{h}$ fulfill $\|v\|_{\infty, \Omega}+\left\|v_{h}\right\|_{\infty, \Omega} \leq M$, and suppose that $y_{v}$ and $y_{h}\left(v_{h}\right)$ are the solutions of (2.1) and (4.2) corresponding to $v$ and $v_{h}$ respectively. Moreover, let $\varphi_{j v}$ and $\varphi_{h j}\left(v_{h}\right)$ be the solutions of (2.6) and (4.7) if $j=0$ and (2.9) and (4.10) if $1 \leq j \leq n_{e}+n_{i}$ corresponding to $v$ and $v_{h}$ respectively. Then the following estimates hold for every $0 \leq j \leq n_{e}+n_{i}$

$$
\begin{aligned}
\left\|y_{v}-y_{h}\left(v_{h}\right)\right\|_{H^{1}(\Omega)}+\left\|\varphi_{j v}-\varphi_{h j}\left(v_{h}\right)\right\|_{H^{1}(\Omega)} & \leq C\left(h+\left\|v-v_{h}\right\|_{L^{2}(\Omega)}\right), \\
\left\|y_{v}-y_{h}\left(v_{h}\right)\right\|_{L^{2}(\Omega)}+\left\|\varphi_{j v}-\varphi_{h j}\left(v_{h}\right)\right\|_{L^{2}(\Omega)} & \leq C\left(h^{2}+\left\|v-v_{h}\right\|_{L^{2}(\Omega)}\right), \\
\left\|y_{v}-y_{h}\left(v_{h}\right)\right\|_{L^{\infty}(\Omega)}+\left\|\varphi_{j v}-\varphi_{h j}\left(v_{h}\right)\right\|_{L^{\infty}(\Omega)} & \leq C\left(h^{\sigma}+\left\|v-v_{h}\right\|_{L^{2}(\Omega)}\right),
\end{aligned}
$$

where $C \equiv C(\Omega, n, M)$ is a positive constant independent of $h$, and $\sigma=1$ if the triangulation is of nonnegative type or $\sigma=2-n / 2$ in other case.

The reader is referred to Ciarlet [12] for the definition and properties of triangulations of nonnegative type.

Lemma 4.5. Let $u_{h} \rightarrow u$ weakly in $L^{1}(\Omega)$, with $\alpha \leq u_{h} \leq \beta$ for every $h>0$, then $y_{h}\left(u_{h}\right) \rightarrow y_{u}$ and $\varphi_{h j}\left(u_{h}\right) \rightarrow \varphi_{j u}$ in $H_{0}^{1}(\Omega) \cap C(\bar{\Omega})$ strongly for every $0 \leq j \leq n_{e}+n_{i}$. Moreover $J(u) \leq \liminf _{h \rightarrow 0} J_{h}\left(u_{h}\right)$.

The next theorem establishes that $U_{\text {had }}$ is non empty for every $h$ small enough and that the regular controls $\bar{u}$ can be approximated by elements of $U_{\text {had }}$.

Theorem 4.6. Let us assume that $\bar{u} \in C^{0,1}(\bar{\Omega})$ is a feasible control of problem $(\mathrm{P})$ for which (3.1) holds. Then there exist $h_{0}>0$, a sequence $\left\{u_{h}\right\}_{0<h<h_{0}}$, with $u_{h} \in U_{\text {had }}$, and a constant $C=C\left(\Omega, n,\|\bar{u}\|_{C^{0,1}(\bar{\Omega})}\right)$ such that

$$
\left\|\bar{u}-u_{h}\right\|_{L^{\infty}(\Omega)} \leq C h^{\sigma},
$$

where $\sigma$ is as in Lemma 4.4 .

We state two lemmas before proving this theorem.

Lemma 4.7. Let $\bar{u} \in C^{0,1}(\bar{\Omega})$ and let $\{\bar{w}\}_{j \in I_{0}}$ be given by (3.1). Then there exists a family $\left\{w_{h j}\right\}_{j \in I_{0}} \subset U_{h}$ uniformly bounded in $L^{\infty}(\Omega)$, with $\operatorname{supp}\left(w_{h j}\right) \subset \Omega_{\varepsilon_{\bar{u}} / 2}$ for $h \leq h_{1}$, such that $w_{h j} \rightarrow \bar{w}_{j}$ in $L^{r}(\Omega)$ for every $1 \leq r<+\infty$. Moreover if the functions $\{\bar{w}\}_{j \in I_{0}} \subset C^{0,1}(\bar{\Omega})$, then there exists $C=C(\Omega, n)$ such that

$$
\left\|\bar{w}_{j}-\bar{w}_{h j}\right\|_{L^{\infty}(\Omega)} \leq C h\left\|\bar{w}_{j}\right\|_{C^{0,1}(\bar{\Omega})} \quad \forall j \in I_{0} .
$$

Proof. Let us define $\bar{w}_{h j}=\Pi_{h} \bar{w}_{j}$. It is well known that $\bar{w}_{h j} \rightarrow \bar{w}_{j}$ in $L^{1}(\Omega)$ when $h \rightarrow 0$. Moreover it is obvious that $\left\{\bar{w}_{h j}\right\}_{j \in I_{0}}$ is uniformly bounded in $L^{\infty}(\Omega)$, then $\bar{w}_{h j} \rightarrow \bar{w}_{j}$ in $L^{r}(\Omega)$ for every $1 \leq r<\infty$. Since $\bar{u}$ is Lipschitz in $\bar{\Omega}$, then there exists $\bar{c}>0$ such that $\left|\bar{u}\left(x_{2}\right)-\bar{u}\left(x_{1}\right)\right| \leq \bar{c}\left|x_{2}-x_{1}\right|$ for every $x_{2}, x_{1} \in \bar{\Omega}$. Let us take $h_{1}>0$ such that $\bar{c} \max \left\{\rho(T): T \in \tilde{\mathcal{T}}_{h}\right\}<\varepsilon_{\bar{u}} / 2$ for $h \leq h_{1}$. 
Let $x \in \Omega$ such that $\bar{u}(x)>\beta-\bar{\varepsilon}_{\bar{u}} / 2$ and let $T \in \tilde{\mathcal{T}}_{h}$, with $h \leq h_{1}$, such that $x \in T$. For any $x^{\prime} \in T$ we have

$$
\bar{u}\left(x^{\prime}\right)=\bar{u}(x)+\left(\bar{u}\left(x^{\prime}\right)-\bar{u}(x)\right)>\beta-\varepsilon_{\bar{u}} / 2-\bar{c}\left|x^{\prime}-x\right| \geq \beta-\varepsilon_{\bar{u}} / 2-\bar{c} \rho(T) \geq \beta-\varepsilon_{\bar{u}},
$$

therefore $\bar{w}_{j}\left(x^{\prime}\right)=0$ for every $x^{\prime} \in T$ and every $j \in I_{0}$, consequently $\bar{w}_{h j \mid T}=0$, in particular $\bar{w}_{h j}(x)=0$ for every $j \in I_{0}$.

Analogously we can prove that if $\bar{u}(x)<\alpha+\bar{\varepsilon}_{\bar{u}} / 2$, then $w_{h j}(x)=0$ for all $h \leq h_{1}$ and every $j \in I_{0}$.

Finally, if $\bar{w}_{j} \in C^{0,1}(\bar{\Omega})$, then it is well known [12] that

$$
\left\|\bar{w}_{j}-\Pi_{h} \bar{w}_{j}\right\|_{L^{\infty}(\Omega)} \leq C h\left\|\bar{w}_{j}\right\|_{C^{0,1}(\bar{\Omega})}
$$

which concludes the proof.

Lemma 4.8. Let $\bar{u} \in C^{0,1}(\bar{\Omega})$ be a feasible and regular control of $(\mathrm{P})$ and let $u_{h} \in U_{h}$ such that $\alpha \leq u_{h} \leq \beta$ and $u_{h} \rightarrow \bar{u}$ in $L^{p}(\Omega)$. Then there exist $h_{2}>0$ and $\left\{\bar{w}_{h j}\right\}_{j \in I_{0}}$ such that $\bar{w}_{h j} \rightarrow \bar{w}_{j}$ in $L^{p}(\Omega), \operatorname{supp}\left(\bar{w}_{h j}\right) \subset \Omega_{\varepsilon_{\bar{u}} / 2}$ $\cap \Omega_{h, \varepsilon_{\bar{u}} / 4}$ for all $j \in I_{0}$ and $h \leq h_{2}$, and $G_{h i}^{\prime}\left(u_{h}\right) \bar{w}_{h j}=\delta_{i j}, i, j \in I_{0}$, where

$$
\Omega_{h, \varepsilon_{\bar{u}} / 4}=\left\{x \in \Omega: \alpha+\varepsilon_{\bar{u}} / 4 \leq u_{h}(x) \leq \beta-\varepsilon_{\bar{u}} / 4\right\} .
$$

Moreover if $u_{h} \rightarrow \bar{u}$ in $L^{\infty}(\Omega)$, then we can take $\left\{\bar{w}_{h j}\right\}_{j \in I_{0}} \subset U_{h}$ in such a way that

$$
\left\|\bar{w}_{j}-\bar{w}_{h j}\right\|_{L^{\infty}(\Omega)} \leq C\left\{h+\left\|\bar{u}-u_{h}\right\|_{L^{2}(\Omega)}\right\} \quad \forall j \in I_{0} .
$$

Proof. Let $\left\{w_{h j}\right\}_{j \in I_{0}}$ be the family obtained in Lemma 4.7. We set for every $j \in I_{0}$

$$
\tilde{w}_{h j}(x)=\left\{\begin{array}{cl}
w_{h j}(x) & \text { if } x \in \Omega_{h, \varepsilon_{\bar{u}} / 4} \\
0 & \text { otherwise }
\end{array}\right.
$$

Then the support of each $\tilde{w}_{h j}$ is contained in $\Omega_{\varepsilon_{\bar{u}} / 2} \cap \Omega_{h, \varepsilon_{\bar{u} / 4}}$ as required. It is clear that

$$
\operatorname{supp}\left(\tilde{w}_{h j}-w_{h j}\right) \subset \Omega_{\varepsilon_{\bar{u}} / 2} \cap\left[\Omega \backslash \Omega_{h, \varepsilon_{\bar{u}} / 4}\right] \subset\left\{x \in \Omega:\left|\bar{u}(x)-\bar{u}_{h}(x)\right|>\frac{\varepsilon_{\bar{u}}}{4}\right\}=X_{h} .
$$

Since $u_{h} \rightarrow \bar{u}$ in $L^{p}(\Omega)$, we have that the Lebesgue measure of $X_{h}$ tends to zero when $h \rightarrow 0$. On the other hand, $\left\{w_{h j}\right\}_{h>0}$ is uniformly bounded in $L^{\infty}(\Omega)$, therefore $\left(\tilde{w}_{h j}-w_{h j}\right) \rightarrow 0$ in $L^{r}(\Omega)$ for all $r<+\infty$. Consequently for every $j \in I_{0} \tilde{w}_{h j} \rightarrow \bar{w}_{j}$ in $L^{r}(\Omega)$ too.

Since $u_{h} \rightarrow \bar{u}$ in $L^{p}(\Omega)$, from Lemma 4.5 it comes that $y_{h}\left(u_{h}\right) \rightarrow \bar{y}=y_{\bar{u}}$ and $\varphi_{h}\left(u_{h}\right) \rightarrow \bar{\varphi}=\varphi_{\bar{u}}$ in $H^{1}(\Omega) \cap C(\bar{\Omega})$. Now from (4.8) we have that $G_{h i}^{\prime}\left(u_{h}\right) \tilde{w}_{h j} \rightarrow G_{i}^{\prime}(\bar{u}) \bar{w}_{j}=\delta_{i j}, i, j \in I_{0}$. We can argue as in the proof of Lemma 3.1 to deduce the existence of $h_{2}>0$ such that the linear mapping $S_{h}: \mathbb{R}^{m} \longrightarrow \mathbb{R}^{m}$ defined by

$$
S_{h}(c)=\left(G_{h i}^{\prime}\left(u_{h}\right)\left[\sum_{j \in I_{0}} c_{j} \tilde{w}_{h j}\right]\right)_{i \in I_{0}}
$$

is an isomorphism for every $h \leq h_{2}$. Therefore if we denote by $\left\{e_{j}\right\}_{j \in I_{0}}$ the canonical base of $\mathbb{R}^{m}$, then we deduce the existence of vectors $c_{h}^{i}=\left(c_{h j}^{i}\right)_{j \in I_{0}}$ such that $S_{h}\left(c_{h}^{i}\right)=e_{i}$. Now setting

$$
\bar{w}_{h i}=\sum_{j \in I_{0}} c_{h j}^{i} \tilde{w}_{h j}
$$


we have that $G_{h i}^{\prime}\left(u_{h}\right) \bar{w}_{h j}=\delta_{i j}$ and using the convergence $S_{h} \rightarrow$ Identity we deduce that

$$
c_{h}^{i}=S_{h}^{-1}\left(e_{i}\right) \rightarrow e_{i},
$$

which proves that $\bar{w}_{h j} \rightarrow \bar{w}_{j}$, for every $j \in I_{0}$. Thus $\left\{\bar{w}_{h j}\right\}_{j \in I_{0}}$ satisfies the requirements of the lemma.

Let us prove the last part of Lemma. Since $\bar{u} \in C(\bar{\Omega})$, by using Lemma 3.1, we can assume that $\left\{\bar{w}_{j}\right\}_{j \in I_{0}}$ $\subset C^{0,1}(\bar{\Omega})$ in (3.1). The convergence $u_{h} \rightarrow \bar{u}$ in $L^{\infty}(\Omega)$ implies that $\Omega_{\varepsilon_{\bar{u}} / 2} \subset \Omega_{h, \varepsilon_{\bar{u}} / 4}$ for $h$ small enough, therefore $\operatorname{supp}\left(\tilde{w}_{h j}\right) \subset \Omega_{\varepsilon_{\bar{u}} / 2}=\Omega_{\varepsilon_{\bar{u}} / 2} \cap \Omega_{h, \varepsilon_{\bar{u}} / 4}$ and $\tilde{w}_{h j}=w_{h j}$. On the other hand, taking into account Lemma 4.4, we have that

$$
\left|G_{i}^{\prime}(\bar{u}) v_{h}-G_{h i}^{\prime}\left(u_{h}\right) v_{h}\right| \leq \int_{\Omega}\left|\varphi_{i \bar{u}}-\varphi_{h i}\left(u_{h}\right)\right|\left|v_{h}\right| \mathrm{d} x \leq C\left(h+\left\|\bar{u}-u_{h}\right\|_{L^{2}(\Omega)}\right)\left\|v_{h}\right\|_{L^{2}(\Omega)} .
$$

From this inequality and (4.15) we get

$$
\begin{aligned}
\left|G_{i}^{\prime}(\bar{u}) \bar{w}_{j}-G_{h i}^{\prime}\left(u_{h}\right) \tilde{w}_{h j}\right| & \leq\left|G_{i}^{\prime}(\bar{u})\left(\bar{w}_{j}-\tilde{w}_{h j}\right)\right|+\left|G_{i}^{\prime}(\bar{u}) \tilde{w}_{h j}-G_{h i}^{\prime}\left(u_{h}\right) \tilde{w}_{h j}\right| \\
& \leq C\left(h+\left\|\bar{u}-u_{h}\right\|_{L^{2}(\Omega)}\right) \| .
\end{aligned}
$$

If we set $S(c)=\left(G_{i}^{\prime}(\bar{u})\left[\sum_{j \in I_{0}} c_{j} \bar{w}_{j}\right]\right)_{i \in I_{0}}$, then (3.1) implies that $S$ coincides with the identity in $\mathbb{R}^{m}$. Therefore from the above inequality we deduce

$$
\| S_{h}-\text { Identity } \| \leq C\left(h+\left\|\bar{u}-u_{h}\right\|_{L^{2}(\Omega)}\right) .
$$

From here we deduce that

$$
\left\|c_{h}^{i}-e_{i}\right\| \leq C^{\prime}\left(h+\left\|\bar{u}-u_{h}\right\|_{L^{2}(\Omega)}\right) .
$$

This estimate along with Lemma 4.7 lead to

$$
\begin{aligned}
\left\|\bar{w}_{i}-\bar{w}_{h i}\right\|_{L^{\infty}(\Omega)} \leq & \left|1-c_{h i}^{i}\right|\left\|\bar{w}_{i}\right\|_{L^{\infty}(\Omega)}+\left|c_{h i}^{i}\right|\left\|\bar{w}_{i}-\tilde{w}_{h i}\right\|_{L^{\infty}(\Omega)} \\
& +\sum_{j \neq i, j \in I_{0}}\left|c_{h j}^{i}\right|\left\|\tilde{w}_{h j}\right\|_{L^{\infty}(\Omega)} \leq C\left(h+\left\|\bar{u}-u_{h}\right\|_{L^{2}(\Omega)}\right) .
\end{aligned}
$$

Proof of Theorem 4.6. Let us apply Lemma 4.8 to the functions $u_{h}=\Pi_{h} \bar{u}$. Let us take $h_{3}>0$ satisfying that $h_{3} \leq \min \left\{h_{1}, h_{2}\right\}$, with $h_{1}$ and $h_{2}$ given in Lemmas 4.7 and 4.8 respectively, and such that $\bar{c} \max \{\rho(T)$ : $\left.T \in \hat{\mathcal{T}}_{h}\right\}<\varepsilon_{\bar{u}} / 4$ for every $h \leq h_{3}$. Since

$$
\bar{w}_{h j}(x)=0 \text { if } \Pi_{h} \bar{u}(x)<\alpha+\varepsilon_{\bar{u}} / 4 \text { or } \Pi_{h} \bar{u}(x)>\beta-\varepsilon_{\bar{u}} / 4
$$

we deduce the existence of $\varepsilon>0$ such that

$$
\alpha \leq \Pi_{h} \bar{u}+\sum_{j \in I_{0}} c_{j} \bar{w}_{h j} \leq \beta \quad \forall x \in \Omega, \quad c \in B_{\varepsilon}(0) \text { and } h \leq h_{3},
$$

where $B_{\varepsilon}(0)$ is the ball of $\mathbb{R}^{m}$ of center 0 and radius $\varepsilon$ and $c=\left(c_{j}\right)_{j \in I_{0}}, m$ being the cardinal of $I_{0}$.

Let us consider the functions $\Psi, \Psi_{h}: \mathbb{R}^{m} \longrightarrow \mathbb{R}^{m}$ defined by

$$
\Psi(c)=\left(G_{i}\left(\bar{u}+\sum_{j \in I_{0}} c_{j} \bar{w}_{j}\right)\right)_{i \in I_{0}} \text { and } \Psi_{h}(c)=\left(G_{h i}\left(\Pi_{h} \bar{u}+\sum_{j \in I_{0}} c_{j} \bar{w}_{h j}\right)\right)_{i \in I_{0}} .
$$


It is immediate that $\Psi_{h} \rightarrow \Psi$ and $D \Psi_{h} \rightarrow D \Psi$ uniformly over compact subsets of $\mathbb{R}^{m}$. Furthermore $D \Psi(0)$ $=$ Identity, then taking $\varepsilon$ sufficiently small we can assume that $\Psi: B_{\varepsilon}(0) \longrightarrow \Psi\left(B_{\varepsilon}(0)\right)$ is a diffeomorphism. Therefore $\Psi_{h}: B_{\varepsilon / 2}(0) \longrightarrow \Psi_{h}\left(B_{\varepsilon / 2}(0)\right)$ is also a diffeomorphism if $h<h_{4}<h_{3}$ for some $h_{4}$ small enough. From the definition of $I_{0}$ and $\Psi$ we know that $\Psi(0)=0$, then it is easy to deduce that there exists a unique $c_{h} \in B_{\varepsilon / 2}(0)$ such that $\Psi_{h}\left(c_{h}\right)=0$ for every $h<h_{5} \leq h_{4}$, with $h_{5}>0$ small enough. Moreover we have that $c_{h} \rightarrow 0$. This along with (4.17) imply that

$$
u_{h}=\Pi_{h} \bar{u}+\sum_{j \in I_{0}} c_{h j} \bar{w}_{h j}
$$

belongs to $U_{\text {had }}$ for every $h<h_{0}$, assumed that $0<h_{0}<h_{5}$ has been chosen in such a way that $G_{h i}\left(u_{h}\right)<0$ for every $j \notin I_{0}$ and $h<h_{0}$, which is obviously possible.

Finally, let us prove the estimate (4.14). By using Lemma 3.1, we can assume that $\left\{\bar{w}_{j}\right\}_{j \in I_{0}} \subset C^{0,1}(\bar{\Omega})$, then Lemma 4.8 applied to the sequence $\left\{\Pi_{h} \bar{u}\right\}_{h>0}$ implies that

$$
\left\|\bar{w}_{j}-\bar{w}_{h j}\right\|_{L^{\infty}(\Omega)} \leq C\left\{h+\left\|\bar{u}-\Pi_{h} \bar{u}\right\|_{L^{2}(\Omega)}\right\} \leq C h \quad \forall j \in I_{0} .
$$

Using the definition of $u_{h}$ (4.18), it is clear that (4.14) follows from the estimates

$$
\left|c_{h j}\right| \leq C h^{\sigma} \quad \forall j \in I_{0}
$$

In order to prove these estimates, we first notice that $(2.7,4.8,4.19,4.11)$ and $(4.13)$ lead to

$$
\sup _{c \in \bar{B}_{r}(0)}\left\|D \Psi(c)-D \Psi_{h}(c)\right\| \leq C_{r} h \quad \text { and } \sup _{c \in \bar{B}_{r}(0)}\left\|\Psi(c)-\Psi_{h}(c)\right\| \leq C_{r} h^{\sigma}
$$

for every $r>0$. Let us take $r \leq \varepsilon / 2$. Second relation of (4.20) implies that

$$
\left\|\Psi_{h}(0)\right\|=\left\|\Psi_{h}(0)-\Psi(0)\right\| \leq C h^{\sigma} .
$$

On the other hand, for every $i \in I_{0}$ we apply the mean value theorem

$$
\Psi_{h i}(0)=\Psi_{h i}(0)-\Psi_{h i}\left(c_{h}\right)=-D \Psi_{h i}\left(\theta_{h i} c_{h}\right) c_{h}
$$

Now defining $M_{h}$ as the matrix having the rows $D \Psi_{h i}\left(\theta_{h i} c_{h}\right)$, we deduce that $M_{h}$ is invertible for $h$ small enough and $M_{h} \rightarrow$ Identity when $h \rightarrow 0$. Therefore $M_{h}^{T} M_{h}$ is a symmetric positive definite matrix with a minimum eigenvalue $\mu_{h} \rightarrow 1$. Then (4.22) and (4.21) lead to

$$
\mu_{h}\left\|c_{h}\right\|^{2} \leq c_{h}^{T} M_{h}^{T} M_{h} c_{h}=\left\|\Psi_{h}(0)\right\|^{2} \leq C h^{2 \sigma},
$$

which proves $\left\|c_{h}\right\| \leq C h^{\sigma}$ as required.

As an immediate consequence of Theorem 4.6 we get that if $(\mathrm{P})$ has a regular control $\bar{u} \in C^{0,1}(\bar{\Omega})$, then $\left(\mathrm{P}_{h}\right)$ has feasible controls for every $h$ small enough and consequently $\left(\mathrm{P}_{h}\right)$ has at least one solution because $U_{\text {had }}$ is a nonempty compact set and $J_{h}$ is continuous in $U_{h}$.

Analogously to (3.1), we will say that a discrete control $\bar{u}_{h} \in U_{\text {had }}$ is regular if

$$
\left\{\begin{array}{l}
\exists \varepsilon_{\bar{u}_{h}}>0 \text { and }\left\{\bar{w}_{h j}\right\}_{j \in I_{0}} \subset U_{h} \text { such that } \\
\operatorname{supp} \bar{w}_{h j} \subset \Omega_{\varepsilon_{\bar{u}_{h}}} \text { and } G_{h i}^{\prime}\left(\bar{u}_{h}\right) \bar{w}_{h j}=\delta_{i j}, \quad i, j \in I_{h 0},
\end{array}\right.
$$

where

$$
I_{h 0}=\left\{j \leq n_{e}+n_{i} \mid G_{h j}\left(\bar{u}_{h}\right)=0\right\}
$$


Lemma 4.8 states that if $\bar{u} \in C^{0,1}(\bar{\Omega})$ is a regular feasible control of $(\mathrm{P})$ and $u_{h} \rightarrow \bar{u}$ in $L^{p}(\Omega)$, then $u_{h}$ is a regular control of $\left(\mathrm{P}_{h}\right)$ for every $h$ small enough. Indeed it is sufficient to notice that the convergence $u_{h} \rightarrow \bar{u}$ implies that $I_{h 0} \subset I_{0}$ for every $h$ small enough and to extract the functions $\left\{\bar{w}_{h j}\right\}_{j \in I_{0 h}}$ from the family provided by Lemma 4.8. The next theorem summarizes the results obtained in this section.

Theorem 4.9. If $\bar{u} \in C^{0,1}(\bar{\Omega})$ is a feasible and regular control for problem $(\mathrm{P})$, then there exist $h_{0}>0$ and controls $\left\{\bar{u}_{h}\right\}_{h<h_{0}}$ such that $\bar{u}_{h} \in U_{\text {had }}$ is a regular control for problem $\left(\mathrm{P}_{h}\right)$ and (4.14) holds. Moreover $\left(\mathrm{P}_{h}\right)$ has at least one solution for every $h<h_{0}$.

It is easy to prove that the existence of a feasible regular control $\bar{u} \in C(\bar{\Omega})$ implies the existence of feasible regular controls in $C^{0,1}(\bar{\Omega})$ close to $\bar{u}$.

\section{Characterization of solutions of $\left(\mathrm{P}_{h}\right)$}

The aim of this section is to characterize the solutions of problem $\left(\mathrm{P}_{h}\right)$ similarly as we did in Section 3 for problem $(\mathrm{P})$.

In the rest of the section $\bar{u}_{h}$ will denote a local solution of $\left(\mathrm{P}_{h}\right)$ which is regular in the sense of (4.23). We define the Lagrangian function associated with $\left(\mathrm{P}_{h}\right)$ by

$$
\mathcal{L}_{h}(u, \lambda)=J_{h}(u)+\sum_{j=1}^{n_{e}+n_{i}} \lambda_{j} G_{h j}(u) .
$$

Analogously to Section 2 we have the following results:

Theorem 5.1. Let us assume that $\bar{u}_{h}$ is a local solution of $\left(\mathrm{P}_{h}\right)$ and (4.23) holds. Then there exist real numbers $\left\{\bar{\lambda}_{h j}\right\}_{j=1}^{n_{e}+n_{i}}$ such that

$$
\begin{gathered}
\bar{\lambda}_{h j} \geq 0 \quad \text { and } \bar{\lambda}_{h j} G_{h j}\left(\bar{u}_{h}\right)=0, \quad \text { if } n_{e}+1 \leq j \leq n_{e}+n_{i} \\
\frac{\partial \mathcal{L}_{h}}{\partial u_{h}}\left(\bar{u}_{h}, \bar{\lambda}_{h}\right)\left(u_{h}-\bar{u}_{h}\right) \geq 0 \quad \text { for all } \alpha \leq u_{h} \leq \beta
\end{gathered}
$$

Denoting by $\bar{\varphi}_{h 0}$ and $\bar{\varphi}_{h j}$ the solutions of (4.7) and (4.10) corresponding to $\bar{u}_{h}$ and setting

$$
\bar{\varphi}_{h}=\bar{\varphi}_{h 0}+\sum_{j=1}^{n_{e}+n_{i}} \bar{\lambda}_{h j} \bar{\varphi}_{h j}
$$

we deduce from Theorems 4.2 and 4.3 and the definition of $\mathcal{L}_{h}$ that

$$
\begin{aligned}
\frac{\partial \mathcal{L}_{h}}{\partial u_{h}}\left(\bar{u}_{h}, \bar{\lambda}_{h}\right) v_{h} & =\int_{\Omega_{h}}\left(\frac{\partial L}{\partial u}\left(x, \bar{y}_{h}, \bar{u}_{h}\right)+\bar{\varphi}_{h 0}\right) v_{h} \mathrm{~d} x+\sum_{j=1}^{n_{e}+n_{i}} \bar{\lambda}_{h j} \int_{\Omega_{h}} \bar{\varphi}_{h j} v_{h} \mathrm{~d} x \\
& =\int_{\Omega_{h}}\left(\frac{\partial L}{\partial u}\left(x, \bar{y}_{h}, \bar{u}_{h}\right)+\bar{\varphi}_{h}\right) v_{h} \mathrm{~d} x=\int_{\Omega_{h}} \mathrm{~d}_{h}(x) v_{h}(x) \forall v_{h} \in U_{h},
\end{aligned}
$$

where $\bar{y}_{h}=G_{h}\left(\bar{u}_{h}\right)=y_{h}\left(\bar{u}_{h}\right)$ and

$$
\mathrm{d}_{h}(x)=\frac{\partial L}{\partial u}\left(x, \bar{y}_{h}(x), \bar{u}_{h}(x)\right)+\bar{\varphi}_{h}(x) .
$$


From (5.2) we deduce that

$$
\int_{T} \mathrm{~d}_{h}(x) \mathrm{d} x=\left\{\begin{array}{cl}
0 & \text { if } \alpha<\bar{u}_{h \mid T}<\beta \\
\geq 0 & \text { if } \bar{u}_{h \mid T}=\alpha \\
\leq 0 & \text { if } \bar{u}_{h \mid T}=\beta
\end{array}\right.
$$

for every $T \in \mathcal{T}_{h}$.

Remark 5.2. From $(5.2,5.5)$ and assumption (4.23) we get

$$
\int_{\Omega_{h}}\left(\frac{\partial L}{\partial u}\left(x, \bar{y}_{h}, \bar{u}_{h}\right)+\bar{\varphi}_{h 0}\right) \bar{w}_{h j} \mathrm{~d} x+\bar{\lambda}_{h j}=\frac{\partial \mathcal{L}}{\partial u_{h}}\left(\bar{u}_{h}, \bar{\lambda}_{h}\right) \bar{w}_{h j}=0
$$

which implies the uniqueness of the Lagrange multipliers provided in Theorem 5.1.

Associated with $\mathrm{d}_{h}$ we set

$$
\mathcal{T}_{h}^{0}=\left\{T \in \mathcal{T}_{h}:\left|\int_{T} \mathrm{~d}_{h}(x) \mathrm{d} x\right|>0\right\}
$$

Given $\left\{\bar{\lambda}_{h j}\right\}_{j=1}^{n_{e}+n_{i}}$ by Theorem 5.1, we define the cone of critical directions

$$
C_{h}^{0}\left(\bar{u}_{h}\right)=\left\{v_{h} \in U_{h} \text { satisfying }(5.9) \text { and } v_{h \mid T}=0 \text { for } T \in \mathcal{T}_{h}^{0}\right\}
$$

with

$$
\left\{\begin{array}{l}
G_{h j}^{\prime}\left(\bar{u}_{h}\right) v_{h}=0 \text { if }\left(j \leq n_{e}\right) \text { or }\left(j>n_{e}, G_{h j}\left(\bar{u}_{h}\right)=0 \text { and } \bar{\lambda}_{h j}>0\right) \\
G_{h j}^{\prime}\left(\bar{u}_{h}\right) v_{h} \leq 0 \text { if }\left(j>n_{e}, G_{h j}\left(\bar{u}_{h}\right)=0 \text { and } \bar{\lambda}_{h j}=0\right) \\
v_{h \mid T}= \begin{cases}\geq 0 & \text { if } \bar{u}_{h \mid T}=\alpha \\
\leq 0 & \text { if } \bar{u}_{h \mid T}=\beta .\end{cases}
\end{array}\right.
$$

Now we are ready to state the second order necessary and sufficient optimality conditions.

Theorem 5.3. Let us assume that $\bar{u}_{h}$ is a local solution of $\left(\mathrm{P}_{h}\right)$, equation (4.23) holds and $\left\{\bar{\lambda}_{h j}\right\}_{j=1}^{m}$ are the Lagrange multipliers satisfying (5.1) and (5.2). Then the following inequality is satisfied

$$
\frac{\partial^{2} \mathcal{L}_{h}}{\partial u_{h}^{2}}\left(\bar{u}_{h}, \bar{\lambda}_{h}\right) v_{h}^{2} \geq 0 \quad \forall v_{h} \in C_{h}^{0}\left(\bar{u}_{h}\right)
$$

Theorem 5.4. Let $\bar{u}_{h}$ be an admissible control for problem $\left(\mathrm{P}_{h}\right)$ satisfying the regularity assumption (4.23) and (5.1-5.2) for some $\bar{\lambda}_{h j}, j=1, \ldots, n_{i}+n_{e}$. Let us suppose also that

$$
\frac{\partial^{2} \mathcal{L}_{h}}{\partial u_{h}^{2}}\left(\bar{u}_{h}, \bar{\lambda}_{h}\right) v_{h}^{2}>0 \text { for all } v \in C_{h}^{0}\left(\bar{u}_{h}\right) \backslash\{0\} .
$$

Then there exist $\bar{\varepsilon}_{h}>0$ and $\bar{\mu}_{h}>0$ such that $J_{h}\left(\bar{u}_{h}\right)+\bar{\mu}_{h}\left\|u_{h}-\bar{u}_{h}\right\|_{L^{2}(\Omega)}^{2} \leq J_{h}\left(u_{h}\right)$ for all admissible control $u_{h}$ with $\left\|u_{h}-\bar{u}_{h}\right\|_{L^{\infty}(\Omega)} \leq \bar{\varepsilon}_{h}$.

We finish this section with a result analogous to Theorem 3.7. 
Theorem 5.5. Suppose that $\bar{u}_{h}$ is a local solution of $\left(\mathrm{P}_{h}\right)$ and assumptions (A1-A3) and (4.23) are satisfied. Then, for all $T \in \mathcal{T}_{h}$, the equation

$$
\int_{T}\left[\varphi_{h}\left(\bar{u}_{h}\right)(x)+\frac{\partial L}{\partial u}\left(x, y_{h}\left(\bar{u}_{h}\right)(x), t\right)\right] \mathrm{d} x=0,
$$

has a unique solution $\bar{t}=\bar{s}_{T}$. The mapping $\bar{s}_{h} \in U_{h}$, defined by $\bar{s}_{h \mid T}=\bar{s}_{T}$, is related to $\bar{u}_{h}$ by the formula

$$
\bar{u}_{h}(x)=\operatorname{Proj}_{[\alpha, \beta]}\left(\bar{s}_{h}(x)\right)=\max \left(\alpha, \min \left(\beta, \bar{s}_{h}(x)\right)\right) .
$$

\section{Convergence Results}

In this section we will prove that the solutions of discrete problems $\left(\mathrm{P}_{h}\right)$ converge strongly in $L^{2}(\Omega)$ and $L^{\infty}(\Omega)$ to solutions of problem $(\mathrm{P})$. Also we will see that any regular local minimum of $(\mathrm{P})$ satisfying the sufficient optimality conditions can be approximated by regular local minima of the problems $\left(\mathrm{P}_{h}\right)$. Finally we study the order of the approximations of these regular local minima. Now we have the first result of the section.

Theorem 6.1. Let us assume that $(\mathrm{P})$ has at least one regular solution (in the sense of $(3.1)$ ) and let $\left\{\bar{u}_{h}\right\}_{h>0}$ be any sequence of solutions of $\left(\mathrm{P}_{h}\right)$. Then there exist weakly*-converging subsequences in $L^{\infty}(\Omega)$ (still indexed by $h)$. If the subsequence $\left\{\bar{u}_{h}\right\}_{h>0}$ is converging weakly* to $\bar{u}$, then $\bar{u}$ is a solution of $(\mathrm{P})$ and

$$
\lim _{h \rightarrow 0} J_{h}\left(\bar{u}_{h}\right)=J(\bar{u})=\inf (P) \quad \text { and } \quad \lim _{h \rightarrow 0}\left\|\bar{u}-\bar{u}_{h}\right\|_{L^{2}(\Omega)}=0
$$

Furthermore if $\bar{u}$ is a regular control of $(\mathrm{P})$, then there exists $h_{0}>0$ such that $\bar{u}_{h}$ is regular for $\left(\mathrm{P}_{h}\right)$ for each $h<h_{0}$ and

$$
\lim _{h \rightarrow 0}\left\|\bar{u}-\bar{u}_{h}\right\|_{L^{\infty}(\Omega)}=0 \text { and } \lim _{h \rightarrow 0} \bar{\lambda}_{h}=\bar{\lambda}
$$

where $\bar{\lambda}_{h}$ and $\bar{\lambda}$ are the Lagrange multipliers obtained in Theorems 5.1 and 3.2 respectively.

Proof. The existence of subsequences weakly* convergent in $L^{\infty}(\Omega)$ is an obvious consequence of the fact that $-\infty<\alpha \leq \bar{u}_{h} \leq \beta<+\infty$ for every $h$. Any limit point $\bar{u}$ satisfies $\alpha \leq \bar{u} \leq \beta$ and, using Lemma 4.5, $F_{j}\left(y_{\bar{u}}\right)=\lim _{h \rightarrow 0} F_{j}\left(y_{h}\left(\bar{u}_{h}\right)\right)=0$ for every $1 \leq j \leq n_{e}$ and $\leq 0$ for $n_{e}+1 \leq j \leq n_{e}+n_{i}$. Therefore $\bar{u}$ is a feasible control for problem $(\mathrm{P})$. Let $\tilde{u}$ be a regular solution of problem (P). From Theorems 3.7 and 4.6 we obtain a sequence $\left\{u_{h}\right\}_{0<h<h_{0}}$, with $u_{h} \in U_{h a d}$ and $u_{h} \rightarrow \tilde{u}$ in $L^{\infty}(\Omega)$. Then using Lemmas 4.4 and 4.5 along with the fact that $\bar{u}_{h}$ is solution of $\left(\mathrm{P}_{h}\right), u_{h} \in U_{\text {had }}$ and $\bar{u}$ is a feasible control for $(\mathrm{P})$, we get

$$
J(\bar{u}) \leq \liminf _{h \rightarrow 0} J_{h}\left(\bar{u}_{h}\right) \leq \limsup _{h \rightarrow 0} J_{h}\left(\bar{u}_{h}\right) \leq \limsup _{h \rightarrow 0} J_{h}\left(u_{h}\right)=J(\tilde{u})=\inf (\mathrm{P}) \leq J(\bar{u}),
$$

which proves that $\bar{u}$ is a solution of $(\mathrm{P})$ and the first convergence of (6.1). The second limit can be obtained from the hypothesis $\left(\partial^{2} L\right) /\left(\partial u^{2}\right)(x, y, u) \geq \lambda_{L}>0$ assumed in (A2) in the same way than in the proof of [6] (Th. 12).

Let us assume now that $\bar{u}$ is a regular control of $(\mathrm{P})$. The strong convergence of $\left\{\bar{u}_{h}\right\}_{h>0}$ in $L^{2}(\Omega)$ and the uniform boundedness imply the strong convergence in every $L^{r}(\Omega)$ for $r<\infty$. Then the regularity of $\bar{u}_{h}$ follows from Lemma 4.8. Therefore there exist real numbers $\left\{\bar{\lambda}_{h j}\right\}_{j=1}^{n_{e}+n_{i}}$ such that (5.1) and (5.2) hold. From 
Remarks 3.3 and 5.2 and Lemmas 4.4 and 4.8 we deduce that

$$
\begin{aligned}
\lim _{h \rightarrow 0} \bar{\lambda}_{h j} & =-\lim _{h \rightarrow 0} \int_{\Omega_{h}}\left(\frac{\partial L}{\partial u}\left(x, \bar{y}_{h}, \bar{u}_{h}\right)+\bar{\varphi}_{h 0}(x)\right) \bar{w}_{h j}(x) \mathrm{d} x \\
& =-\int_{\Omega}\left(\frac{\partial L}{\partial u}(x, \bar{y}(x), \bar{u}(x))+\bar{\varphi}_{0}(x)\right) \bar{w}_{j}(x) \mathrm{d} x=\bar{\lambda}_{j} .
\end{aligned}
$$

We conclude the proof by establishing the strong convergence of the discrete optimal controls in $L^{\infty}(\Omega)$. Due to Theorems 3.7 and 5.5 , there exist functions $\bar{s} \in C^{0,1}(\bar{\Omega})$ and $\bar{s}_{h} \in U_{h}$ such that

$$
\begin{gathered}
\varphi_{\bar{u}}(x)+\frac{\partial L}{\partial u}\left(x, y_{\bar{u}}(x), \bar{s}(x)\right)=0 \quad \forall x \in \hat{T} \text { and } \forall \hat{T} \in \hat{\mathcal{T}}_{h}, \\
\bar{s}_{h \mid T}=\bar{s}_{T}, \quad \int_{T}\left(\varphi_{h}\left(\bar{u}_{h}\right)+\frac{\partial L}{\partial u}\left(x, \bar{y}_{h}\left(\bar{u}_{h}\right), \bar{s}_{T}\right)\right) \mathrm{d} x=0 \quad \forall T \in \mathcal{T}_{h} .
\end{gathered}
$$

From (6.4), we deduce that for every $T \in \mathcal{T}_{h}$, there exists $x_{T} \in T$ such that

$$
\varphi_{h}\left(\bar{u}_{h}\right)\left(x_{T}\right)+\frac{\partial L}{\partial u}\left(x_{T}, y_{h}\left(\bar{u}_{h}\right)\left(x_{T}\right), \bar{s}_{T}\right)=0
$$

Suppose that $T \in \mathcal{T}_{h}$ is fixed, and select an arbitrary $x \in T$. By making the difference between (6.3) and (6.5), and due to the assumptions made in $\mathbf{A 2}$, it follows that

$$
\begin{aligned}
\lambda_{L}\left|\bar{u}(x)-\bar{u}_{h}(x)\right| & =\lambda_{L}\left|\operatorname{Proj}_{[\alpha, \beta]}(\bar{s}(x))-\operatorname{Proj}_{[\alpha, \beta]}\left(\bar{s}_{h}(x)\right)\right| \leq \lambda_{L}\left|\bar{s}(x)-\bar{s}_{h}(x)\right| \\
& =\lambda_{L}\left|\bar{s}(x)-\bar{s}_{T}\right| \leq\left|\frac{\partial L}{\partial u}\left(x, y_{\bar{u}}(x), \bar{s}(x)\right)-\frac{\partial L}{\partial u}\left(x, y_{\bar{u}}(x), \bar{s}_{T}\right)\right| \\
& =\left|\left(\varphi_{\bar{u}}(x)-\varphi_{h}\left(\bar{u}_{h}\right)\left(x_{T}\right)\right)+\left(\frac{\partial L}{\partial u}\left(x, y_{\bar{u}}(x), \bar{s}_{T}\right)-\frac{\partial L}{\partial u}\left(x_{T}, y_{h}\left(\bar{u}_{h}\right)\left(x_{T}\right), \bar{s}_{T}\right)\right)\right| \\
& \leq\left|\varphi_{\bar{u}}(x)-\varphi_{h}\left(\bar{u}_{h}\right)\left(x_{T}\right)\right|+C\left\{\left|x-x_{T}\right|+\left|y_{\bar{u}}(x)-y_{h}\left(\bar{u}_{h}\right)\left(x_{T}\right)\right|\right\}
\end{aligned}
$$

We know from the regularity $y_{\bar{u}}, \varphi_{\bar{u}} \in W^{2, p}(\Omega)$ that these functions are Lipschitz, hence

$$
\begin{aligned}
\lambda_{L}\left|\bar{u}(x)-\bar{u}_{h}(x)\right| & \leq C\left(\left|x-x_{T}\right|+\left\|\varphi_{\bar{u}}-\varphi_{h}\left(\bar{u}_{h}\right)\right\|_{L^{\infty}(T)}+\left\|y_{\bar{u}}-y_{h}\left(\bar{u}_{h}\right)\right\|_{L^{\infty}(T)}\right) \\
& \leq C\left(h+\left\|\varphi_{\bar{u}}-\varphi_{h}\left(\bar{u}_{h}\right)\right\|_{L^{\infty}(T)}+\left\|y_{\bar{u}}-y_{h}\left(\bar{u}_{h}\right)\right\|_{L^{\infty}(T)}\right) .
\end{aligned}
$$

Invoking Lemma 4.4 , the convergence $\bar{\lambda}_{h} \rightarrow \bar{\lambda}$ and the definitions

$$
\varphi_{\bar{u}}=\varphi_{0}(\bar{u})+\sum_{j=1}^{n_{e}+n_{i}} \bar{\lambda}_{j} \varphi_{j \bar{u}} \quad \text { and } \quad \varphi_{h}\left(\bar{u}_{h}\right)=\varphi_{h 0}(\bar{u})+\sum_{j=1}^{n_{e}+n_{i}} \bar{\lambda}_{h j} \varphi_{h j}\left(\bar{u}_{h}\right),
$$

we deduce

$$
\begin{aligned}
\left\|\bar{u}-\bar{u}_{h}\right\|_{L^{\infty}\left(\Omega_{h}\right)}= & \sup _{T \in \mathcal{T}_{h}}\left\|\bar{u}-\bar{u}_{h}\right\|_{L^{\infty}(T)} \leq C\left(h+\left\|\varphi_{\bar{u}}-\varphi_{h}\left(\bar{u}_{h}\right)\right\|_{L^{\infty}\left(\Omega_{h}\right)}\right. \\
& \left.+\left\|y_{\bar{u}}-y_{h}\left(\bar{u}_{h}\right)\right\|_{L^{\infty}\left(\Omega_{h}\right)}\right) \rightarrow 0 \text { when } h \rightarrow 0
\end{aligned}
$$

Let now take an arbitrary $\hat{T} \in \partial \hat{\mathcal{T}}_{h}$, and let $T \in \partial \mathcal{T}_{h}$ be the corresponding boundary triangle satisfying $\hat{T} \supset T$ (here $\partial \hat{\mathcal{T}}_{h}$ and $\partial \mathcal{T}_{h}$ denote the sets of boundary triangles in $\hat{\mathcal{T}}_{h}$ and $\mathcal{T}_{h}$ ). For $\hat{x} \in \hat{T} \backslash T$ let $x$ be its projection on 
the boundary $\Gamma_{h}$ of $\Omega_{h}$. Taking into account the Lipschitz continuity of $\bar{u}$, we obtain

$$
\begin{aligned}
\left|\bar{u}(\hat{x})-\bar{u}_{h}(\hat{x})\right| & \leq|\bar{u}(\hat{x})-\bar{u}(x)|+\left|\bar{u}(x)-\bar{u}_{h}(\hat{x})\right|=|\bar{u}(\hat{x})-\bar{u}(x)|+\left|\bar{u}(x)-\bar{u}_{h}(x)\right| \\
& \leq \bar{c}|\hat{x}-x|+\left\|\bar{u}-\bar{u}_{h}\right\|_{L^{\infty}\left(\Omega_{h}\right)} \leq \bar{c} h+\left\|\bar{u}-\bar{u}_{h}\right\|_{L^{\infty}\left(\Omega_{h}\right) .}
\end{aligned}
$$

Hence

$$
\left\|\bar{u}-\bar{u}_{h}\right\|_{L^{\infty}\left(\Omega \backslash \Omega_{h}\right)}=\sup _{\hat{T} \in \partial \hat{\mathcal{T}}_{h}}\left\|\bar{u}-\bar{u}_{h}\right\|_{L^{\infty}(\hat{T} \backslash T)} \leq \bar{c} h+\left\|\bar{u}-\bar{u}_{h}\right\|_{L^{\infty}\left(\Omega_{h}\right)} \rightarrow 0,
$$

which completes the proof.

The following theorem proves that the local minima $\bar{u}$ of $(\mathrm{P})$ which are regular and satisfy the sufficient optimality conditions are in somehow attractors. More precisely, there exists a neighbourhood of each one of these points such that the problems $\left(\mathrm{P}_{h}\right)$ have local minima in this neighbourhoods which are regular points of $\left(\mathrm{P}_{h}\right)$ and converge uniformly to $\bar{u}$. Therefore if we solve numerically the discrete problem $\left(\mathrm{P}_{h}\right)$, we can approximate $\bar{u}$ in the $L^{\infty}(\Omega)$ norm if we start the iterations in the mentioned neighbourhood of $\bar{u}$. In the sequel $B_{\rho}(u)$ will denote the $L^{\infty}(\Omega)$-ball of center $u$ and radius $\rho$.

Theorem 6.2. Let $\bar{u}$ be a local minimum of (P) satisfying the regularity condition (3.1) and the sufficient optimality condition (3.13). Then there exist $\rho>0$ and $h_{0}>0$ such that the problem $\left(\mathrm{P}_{h}\right)$ has a local minimum $\bar{u}_{h}$ in $B_{\rho}(\bar{u})$ for every $h<h_{0}$. Furthermore every $\bar{u}_{h}$ is regular in the sense of (4.23) and the convergences $(6.2)$ hold.

Proof. Let $\bar{\varepsilon}>0$ be given by Theorem 3.5 and for every $0<\rho \leq \bar{\varepsilon}$ let us consider the problems

$$
\left(\mathrm{P}_{\rho}\right)\left\{\begin{array}{l}
\min J(u)=\int_{\Omega} L\left(x, y_{u}(x), u(x)\right) \mathrm{d} x \\
\text { subject to }\left(y_{u}, u\right) \in\left(C(\bar{\Omega}) \cap H^{1}(\Omega)\right) \times B_{\rho}(\bar{u}), \\
\alpha \leq u(x) \leq \beta \quad \text { a.e. } \quad x \in \Omega, \\
F_{j}\left(y_{u}\right)=0, \quad 1 \leq j \leq n_{e} \\
F_{j}\left(y_{u}\right) \leq 0, \quad n_{e}+1 \leq j \leq n_{e}+n_{i}
\end{array}\right.
$$

and

$$
\left(\mathrm{P}_{h \rho}\right)\left\{\begin{array}{l}
\min J_{h}\left(u_{h}\right)=\int_{\Omega_{h}} L\left(x, y_{h}\left(u_{h}\right)(x), u_{h}(x)\right) \mathrm{d} x, \\
\text { subject to }\left(y_{h}\left(u_{h}\right), u_{h}\right) \in Y_{h} \times\left(U_{h} \cap \bar{B}_{\rho}(\bar{u})\right), \\
\alpha \leq u_{h}(x) \leq \beta \quad \text { a.e. } \quad x \in \Omega_{h}, \\
F_{j}\left(y_{h}\left(u_{h}\right)\right)=0, \quad 1 \leq j \leq n_{e}, \\
F_{j}\left(y_{h}\left(u_{h}\right) \leq 0, \quad n_{e}+1 \leq j \leq n_{e}+n_{i} .\right.
\end{array}\right.
$$

According to Theorem 3.5, $\bar{u}$ is the unique solution of $\left(\mathrm{P}_{\rho}\right)$. From Theorem 3.7, we know that $\bar{u} \in C^{0,1}(\bar{\Omega})$ and Theorem 4.6 states the existence of $h_{0}>0$ and a sequence $\left\{u_{h}\right\}_{h<h_{0}}$ converging to $\bar{u}$ in $L^{\infty}(\Omega)$ and such that $u_{h} \in U_{\text {had }}$. From the convergence $\left\|\bar{u}-u_{h}\right\|_{L^{\infty}(\Omega)} \rightarrow 0$ we also know that $u_{h} \in B_{\rho}(\bar{u})$ if $h_{0}$ is chosen small enough. Therefore $\left(\mathrm{P}_{h \rho}\right)$ has feasible controls for $h<h_{0}$ and consequently it has at least one solution $\bar{u}_{h}$ for every $h<h_{0}$. We can argue as in the first part of the proof of Theorem 6.1 to deduce that $\left\|\bar{u}-\bar{u}_{h}\right\|_{L^{2}(\Omega)} \rightarrow 0$ when $h \rightarrow 0$. Moreover, since $\left\{\bar{u}_{h}\right\}_{h<h_{0}}$ is uniformly bounded in $L^{\infty}(\Omega)$, then the convergence $\bar{u}_{h} \rightarrow \bar{u}$ holds in $L^{r}(\Omega)$ for all $r<\infty$. Let us see that the convergence is also fulfilled in $L^{\infty}(\Omega)$. 
Let $\left\{\bar{w}_{h j}\right\}_{j \in I_{0}}$ be given by Lemma 4.8 and let us define $\left\{w_{h j}\right\}_{j \in I_{0}}$ as follows

$$
\forall T \in \mathcal{T} \quad w_{h j \mid T}=\left\{\begin{array}{cl}
\bar{w}_{h j \mid T} & \text { if }\left\|\bar{u}-\bar{u}_{h}\right\|_{L^{\infty}(T)}<\rho \\
0 & \text { otherwise. }
\end{array}\right.
$$

Let us set

$$
\Sigma_{h}=\left\{T \in \mathcal{T}:\left\|\bar{u}-\bar{u}_{h}\right\|_{L^{\infty}(T)}=\rho\right\} \quad \text { and } \quad A_{h}=\bigcup_{T \in \Sigma_{h}} T .
$$

If we prove that $\left|A_{h}\right| \rightarrow 0$ when $h \rightarrow 0$, then we will obtain the convergence $\bar{w}_{h j}-w_{h j} \rightarrow 0$ in $L^{r}(\Omega)$ for every $r<+\infty$ and consequently $w_{h j} \rightarrow \bar{w}_{j}$ in $L^{r}(\Omega)$ for every $j \in I_{0}$. Here $\left|A_{h}\right|$ denotes the Lebesgue measure of $A_{h}$. Let $\bar{c}$ be the Lipschitz constant of $\bar{u}$ and let us assume that $h_{0}$ has been chosen satisfying $\bar{c} h_{0}<\rho / 2$. Thus if $h<h_{0}, T \in \Sigma_{h}$ and $x_{T} \in T$ verifies $\left|\bar{u}\left(x_{T}\right)-\bar{u}_{h \mid T}\right|=\rho$, then we have for every $x \in T$

$$
\left|\bar{u}(x)-\bar{u}_{h}(x)\right| \geq\left|\bar{u}\left(x_{T}\right)-\bar{u}_{h \mid T}\right|-\left|\bar{u}\left(x_{T}\right)-\bar{u}(x)\right| \mid \geq \rho-\bar{c} h>\frac{\rho}{2} .
$$

Since $\bar{u}_{h} \rightarrow \bar{u}$ in $L^{1}(\Omega)$, the above inequality implies

$$
\frac{\rho}{2}\left|A_{h}\right| \leq \int_{A_{h}}\left|\bar{u}(x)-\bar{u}_{h}(x)\right| \mathrm{d} x \leq \int_{\Omega}\left|\bar{u}(x)-\bar{u}_{h}(x)\right| \mathrm{d} x \rightarrow 0
$$

as required.

Now we can proceed as in the proof of Lemma 3.1 to deduce the existence of a family $\left\{\tilde{w}_{h j}\right\}_{j \in I_{0}}$ such that $G_{i}\left(\bar{u}_{h}\right) \tilde{w}_{h j}=\delta_{i j}$, supp $\tilde{w}_{h j} \subset \Omega_{h \varepsilon} \cap\left(\Omega \backslash A_{h}\right)$ for some $\varepsilon>0$ and $\tilde{w}_{h j} \rightarrow \bar{w}_{j}$ for every $j \in I_{0}$. Since $F_{j}\left(y_{h}\left(\bar{u}_{h}\right)\right) \rightarrow F_{j}\left(y_{\bar{u}}\right)$, then $I_{h 0} \subset I_{0}$ for $h<h_{0}$, with $h_{0}$ small enough. Hence $\bar{u}_{h}$ is a regular local minimum for problem $\left(\mathrm{P}_{h \rho}\right)$. Then we can deduce the first order optimality conditions similar to those of Theorem 5.1. So there exist real numbers $\left\{\bar{\lambda}_{h j}\right\}_{j=1}^{n_{e}+n_{i}}$ such that

$$
\begin{gathered}
\bar{\lambda}_{h j} \geq 0 \text { and } \bar{\lambda}_{h j} G_{h j}\left(\bar{u}_{h}\right)=0 \text { if } n_{e}+1 \leq j \leq n_{e}+n_{i}, \\
\frac{\partial \mathcal{L}_{h}}{\partial u_{h}}\left(\bar{u}_{h}, \bar{\lambda}_{h}\right)\left(u_{h}-\bar{u}_{h}\right) \geq 0 \text { for all } \alpha \leq u_{h} \leq \beta \text { and } u_{h} \in \bar{B}_{\rho}(\bar{u}) .
\end{gathered}
$$

Using Remarks 3.3 and 5.2 and arguing as in the proof of the previous theorem, we deduce that $\bar{\lambda}_{h} \rightarrow \bar{\lambda}$. On the other hand, if we denote

$$
u_{\alpha}(x)=\max \{\alpha, \bar{u}(x)-\rho\} \quad \text { and } \quad u_{\beta}(x)=\min \{\beta, \bar{u}(x)+\rho\},
$$

Theorem 5.5 is still valid replacing (5.13) by

$$
\bar{u}_{h}(x)=\operatorname{Proj}_{\left[u_{\alpha}(x), u_{\beta}(x)\right]}\left(\bar{s}_{h}(x)\right)=\max \left(u_{\alpha}(x), \min \left(u_{\beta}(x), \bar{s}_{h}(x)\right)\right) .
$$

Now we can repeat the proof of Theorem 6.1 to deduce that $\left\|\bar{u}-\bar{u}_{h}\right\|_{L^{\infty}(\Omega)} \rightarrow 0$ and therefore $\bar{u}_{h} \in B_{\rho}(\bar{u})$ for every $h$ smaller than a certain $h_{0}$.

In the sequel, $\bar{u}$ will denote a local solution of $(\mathrm{P})$, which is regular in the sense of (3.1) and satisfies the sufficient optimality conditions (3.13). Let $\bar{s} \in C^{0,1}(\bar{\Omega})$ be given by Theorem 3.7. We also have the sequence $\left\{\bar{u}_{h}\right\}_{h<h_{0}}$ of local solutions of $\left(\mathrm{P}_{h}\right)$ provided by Theorem 6.2. Associated to the functions $\left(\bar{s}, \bar{u}, \bar{u}_{h}\right)$ we define

$$
u_{h}(x)=\operatorname{Proj}_{[\alpha, \beta]}\left[\left(\Pi_{h} \bar{s}\right)(x)\right]
$$


and

$$
\hat{u}_{h}(x)= \begin{cases}\bar{u}(x) & \text { if } \bar{u}(x)=\alpha \text { or } \bar{u}(x)=\beta \\ \bar{u}_{h}(x) & \text { if } \alpha<\bar{u}(x)<\beta \text { and } x \in \Omega \backslash \Omega_{h} \\ u_{h}(x) & \text { if } \alpha<\bar{u}(x)<\beta \text { and } x \in \Omega_{h} .\end{cases}
$$

Now we take

$$
\alpha_{h j}=G_{j}^{\prime}(\bar{u})\left(\bar{u}_{h}-\hat{u}_{h}\right) \text { and } \tilde{u}_{h}=\hat{u}_{h}-\sum_{j \in \hat{I}_{h}} \alpha_{h j} \bar{w}_{j}
$$

where $\bar{w}_{j}$ is introduced in (3.1) and

$$
\hat{I}_{h}=\left\{1, \ldots, n_{i}\right\} \cup\left\{j>n_{i}: \bar{\lambda}_{j}>0 \text { or }\left(\bar{\lambda}_{j}=0, j \in I_{0} \text { and } \alpha_{h j}>0\right)\right\} .
$$

Now we deduce the following lemma from Theorem 3.6:

Lemma 6.3. If $h_{0}>0$ is taken small enough, then $\left\{\bar{u}_{h}-\tilde{u}_{h}\right\}_{h<h_{0}} \subset C_{\bar{u}}^{\bar{\tau}}$ for some $\bar{\tau}>0$ and

$$
\frac{\partial^{2} \mathcal{L}}{\partial u^{2}}(\bar{u}, \bar{\lambda})\left(\bar{u}_{h}-\tilde{u}_{h}\right)^{2} \geq \bar{\mu}\left\|\bar{u}_{h}-\tilde{u}_{h}\right\|_{L^{2}(\Omega)}^{2} \quad \forall h<h_{0} .
$$

Proof. Thanks to Theorem 3.6, it is enough to prove that $\bar{u}_{h}-\tilde{u}_{h} \in C_{\bar{u}}^{\bar{\tau}}$ to deduce (6.9). Let us check this inclusion. If $\bar{u}(x)=\alpha$, then $\bar{w}_{j}(x)=0$ for every $j \in I_{0}$, and consequently $\left(\bar{u}_{h}-\tilde{u}_{h}\right)(x)=\bar{u}_{h}(x)-\bar{u}(x)$ $=\bar{u}_{h}(x)-\alpha \geq 0$. Analogously $\left(\bar{u}_{h}-\tilde{u}_{h}\right)(x) \leq 0$ whenever $\bar{u}(x)=\beta$. Therefore $\bar{u}_{h}-\tilde{u}_{h}$ satisfies the sign condition of (3.11). On the other hand, from the definition of $\alpha_{h j}$ and $\tilde{u}_{h}$ and the property of the family $\left\{\bar{w}_{j}\right\}_{j \in I_{0}}$ we conclude that $\left(\bar{u}_{h}-\tilde{u}_{h}\right)$ satisfies all the conditions of (3.11). To conclude the proof we have to prove that $\bar{u}_{h}-\tilde{u}_{h}=0$ in $\Omega^{\bar{\tau}}$. Let us fix $\hat{x} \in \Omega$ and take $\hat{T} \in \hat{\mathcal{T}}_{h}$ such that $\hat{x} \in \hat{T}$. Let us also consider $T \in \mathcal{T}_{h}$ with $T \subset \hat{T}$. From the definitions of $d$ and $d_{h}$ given in (3.6) and (5.4) respectively, Lemma 4.4 and the convergences (6.2), we deduce that $d_{h} \rightarrow d$ uniformly in $\Omega$. Therefore, by taking $h_{0}>0$ small enough and $h<h_{0}$, we have

$$
\left|\mathrm{d}(x)-\mathrm{d}\left(x^{\prime}\right)\right|<\frac{\bar{\tau}}{5} \forall x, x^{\prime} \in \hat{T} \text { and } \forall \hat{T} \in \hat{\mathcal{T}}_{h}
$$

and

$$
\left\|\mathrm{d}-\mathrm{d}_{h}\right\|_{L^{\infty}(\Omega)}<\frac{\bar{\tau}}{5}
$$

Hence, if $d(\hat{x})>\bar{\tau}$, then $d_{h}(\hat{x})>4 \bar{\tau} / 5$ and

$$
\mathrm{d}_{h}(x)=\mathrm{d}_{h}(\hat{x})+\left(\mathrm{d}_{h}(x)-\mathrm{d}(x)\right)+(\mathrm{d}(x)-\mathrm{d}(\hat{x}))+\left(\mathrm{d}(\hat{x})-\mathrm{d}_{h}(\hat{x})\right)>\bar{\tau} / 5 \quad \forall x \in T .
$$

Therefore $\int_{T} \mathrm{~d}_{h}(x) \mathrm{d} x>0$. Using (5.5) we deduce that $\bar{u}_{h}(\hat{x})=\bar{u}_{h \mid T}=\alpha$. Also we have that $\mathrm{d}(\hat{x})>0$ implies $\bar{u}(\hat{x})=\alpha$. From the definition of $\tilde{u}_{h}$, it follows that $\tilde{u}_{h}(\hat{x})=\bar{u}(\hat{x})=\alpha$ too. Collecting all this, we deduce that $\left(\bar{u}_{h}-\tilde{u}_{h}\right)(\hat{x})=0$. Analogously we can argue in the case of $\mathrm{d}(\hat{x})<-\bar{\tau}$ to arrive to the same conclusion, which completes the proof.

The next lemma provides an error estimate for the term $\bar{u}-\tilde{u}_{h}$ : 
Lemma 6.4. For every $h<h_{0}$ the following inequalities hold

$$
\left\|\bar{u}-\tilde{u}_{h}\right\|_{L^{2}(\Omega)} \leq C\left(h^{\sigma}+\left\|\bar{u}-\bar{u}_{h}\right\|_{L^{2}(\Omega)}^{2}\right),
$$

and

$$
\left\|\bar{u}-\tilde{u}_{h}\right\|_{L^{\infty}\left(\Omega_{h}\right)} \leq C\left(h^{\sigma}+\left\|\bar{u}-\bar{u}_{h}\right\|_{L^{2}(\Omega)}^{2}\right)
$$

where $\sigma$ is taken as in Lemma 4.4 .

Proof. Let us start proving a estimate for $\bar{u}-\hat{u}_{h}$. Using the definition of $\hat{u}_{h}$, the representation of $\bar{u}$ provided by (3.16) and the well known interpolation error estimates in the Sobolev spaces, see Ciarlet [12], we get

$$
\begin{aligned}
\left\|\bar{u}-\hat{u}_{h}\right\|_{L^{\infty}\left(\Omega_{h}\right)} & \leq\left\|\bar{u}-u_{h}\right\|_{L^{\infty}\left(\Omega_{h}\right)}=\left\|\operatorname{Proj}_{[\alpha, \beta]}(\bar{s})-\operatorname{Proj}_{[\alpha, \beta]}\left(\Pi_{h} \bar{s}\right)\right\|_{L^{\infty}\left(\Omega_{h}\right)} \\
& \leq\left\|\bar{s}-\Pi_{h} \bar{s}\right\|_{L^{\infty}\left(\Omega_{h}\right)} \leq C h\|\bar{s}\|_{C^{0,1}(\bar{\Omega})} .
\end{aligned}
$$

The same estimate is obviously fulfilled when the $L^{2}\left(\Omega_{h}\right)$-norm is used. On the other hand, from (4.1) we get

$$
\left\|\bar{u}-\hat{u}_{h}\right\|_{L^{2}\left(\Omega \backslash \Omega_{h}\right)} \leq(\beta-\alpha) \sqrt{\left|\Omega \backslash \Omega_{h}\right|} \leq C h .
$$

Inequalities (6.12) and (6.13) lead to

$$
\left\|\bar{u}-\hat{u}_{h}\right\|_{L^{2}(\Omega)} \leq C h .
$$

Let us estimate the terms $\alpha_{h j}$. First of all, let us write

$$
\alpha_{h j}=G_{j}^{\prime}(\bar{u})\left(\bar{u}-\hat{u}_{h}\right)+G_{j}^{\prime}(\bar{u})\left(\bar{u}_{h}-\bar{u}\right) .
$$

From (6.12) and (6.13) we deduce

$$
\left|\alpha_{h j}\right| \leq C\left\|\bar{u}-\hat{u}_{h}\right\|_{L^{2}(\Omega)}+\left|G_{j}^{\prime}(\bar{u})\left(\bar{u}_{h}-\bar{u}\right)\right| \leq C h+\left|G_{j}^{\prime}(\bar{u})\left(\bar{u}_{h}-\bar{u}\right)\right| .
$$

Making a Taylor development we get

$$
G_{j}\left(\bar{u}_{h}\right)=G_{j}(\bar{u})+G_{j}^{\prime}(\bar{u})\left(\bar{u}_{h}-\bar{u}\right)+\frac{1}{2} G_{j}^{\prime \prime}\left(v_{h j}\right)\left(\bar{u}_{h}-\bar{u}\right)^{2},
$$

with $v_{h j}=\bar{u}+\theta_{h j}\left(\bar{u}_{h}-\bar{u}\right)$ for some $0<\theta_{h j}<1$. If $j \in \hat{I}_{h}$ and $j \leq n_{i}$ or $\bar{\lambda}_{j} \neq 0$, then $G_{h j}\left(\bar{u}_{h}\right)=0$ for every $h$ small enough because $\bar{u}_{h}$ is feasible for $\left(\underline{\mathrm{P}}_{h}\right)$ and either the $j$-restriction is an equality or it is an inequality with a positive Lagrange multiplier $\bar{\lambda}_{h j} \rightarrow \bar{\lambda}_{j}$. Using this along with (6.17) it comes

$$
\begin{aligned}
\left|\alpha_{h j}\right| & \leq C h+\left|G_{j}\left(\bar{u}_{h}\right)-\frac{1}{2} G_{j}^{\prime \prime}\left(v_{h j}\right)\left(\bar{u}_{h}-\bar{u}\right)^{2}\right| \leq\left|G_{j}\left(\bar{u}_{h}\right)\right|+C\left(h+\left\|\bar{u}_{h}-\bar{u}\right\|_{L^{2}(\Omega)}^{2}\right) \\
& =\left|G_{j}\left(\bar{u}_{h}\right)-G_{h j}\left(\bar{u}_{h}\right)\right|+C\left(h+\left\|\bar{u}_{h}-\bar{u}\right\|_{L^{2}(\Omega)}^{2}\right) .
\end{aligned}
$$

By using the error estimates in the approximation $y_{h}\left(u_{h}\right)$ of $y_{u_{h}}$, see Casas and Mateos [6], we get

$$
\begin{aligned}
\left|\alpha_{h j}\right| & \leq\left|F_{j}\left(y_{h}\left(u_{h}\right)\right)-F_{j}\left(y_{u_{h}}\right)\right|+C\left(h+\left\|\bar{u}_{h}-\bar{u}\right\|_{L^{2}(\Omega)}^{2}\right) \\
& \leq C\left(h+\left\|y_{h}\left(u_{h}\right)-y_{u_{h}}\right\|_{L^{\infty}(\Omega)}+\left\|\bar{u}_{h}-\bar{u}\right\|_{L^{2}(\Omega)}^{2}\right) \\
& \leq C\left(h^{\sigma}+\left\|\bar{u}_{h}-\bar{u}\right\|_{L^{2}(\Omega)}^{2}\right) .
\end{aligned}
$$


In the case of $j \in \hat{I}_{h}$, with $j>n_{i}, \bar{\lambda}_{j}=0$ and $\alpha_{h j}>0$, we have to distinguish two situations. First we assume that $G_{j}^{\prime}(\bar{u})\left(\bar{u}_{h}-\bar{u}\right) \leq 0$, then (6.14) and (6.15) lead to

$$
\left|\alpha_{h j}\right|=\alpha_{h j} \leq G_{j}^{\prime}(\bar{u})\left(\bar{u}-\hat{u}_{h}\right) \leq C h .
$$

In the second situation we assume that $G_{j}^{\prime}(\bar{u})\left(\bar{u}_{h}-\bar{u}\right)>0$. Since $j>n_{i}$, we have that $G_{h j}\left(\bar{u}_{h}\right) \leq 0$. Using these two facts, the identity $G_{j}(\bar{u})=0$, equations (6.15) and (6.17) obtain

$$
\begin{aligned}
\left|\alpha_{h j}\right| & =\alpha_{h j} \leq C h+G_{j}^{\prime}(\bar{u})\left(\bar{u}_{h}-\bar{u}\right)=C h+G_{j}\left(\bar{u}_{h}\right)-\frac{1}{2} G_{j}^{\prime \prime}\left(v_{h j}\right)\left(\bar{u}_{h}-\bar{u}\right)^{2} \\
& \leq C h+G_{j}\left(\bar{u}_{h}\right)-G_{h j}\left(\bar{u}_{h}\right)-\frac{1}{2} G_{j}^{\prime \prime}\left(v_{h j}\right)\left(\bar{u}_{h}-\bar{u}\right)^{2} \leq C\left(h+\left\|y_{h}\left(u_{h}\right)-y_{u_{h}}\right\|_{L^{\infty}(\Omega)}+\left\|\bar{u}_{h}-\bar{u}\right\|_{L^{2}(\Omega)}^{2}\right) \\
& \leq C\left(h^{\sigma}+\left\|\bar{u}_{h}-\bar{u}\right\|_{L^{2}(\Omega)}^{2}\right) .
\end{aligned}
$$

Collecting the inequalities $(6.18,6.19)$ and $(6.20)$, we deduce that

$$
\left|\alpha_{h j}\right| \leq C\left(h^{\sigma}+\left\|\bar{u}_{h}-\bar{u}\right\|_{L^{2}(\Omega)}^{2}\right)
$$

for every $j \in \hat{I}_{h}$. Finally, from the definition of $\tilde{u}_{h}$, the estimates (6.12) and (6.14) and those ones obtained for $\alpha_{h j}$ we deduce (6.10) and (6.11).

The next two lemmas are required to prove the error estimates for $\bar{u}-\bar{u}_{h}$. Their proof is an exercise which follows easily from the assumptions $(\mathbf{A 1}-\mathbf{A 3})$ along with the expressions for $\left(\partial^{2} \mathcal{L} / \partial u^{2}\right)$ and $\left(\partial^{2} \mathcal{L}_{h} / \partial u_{h}^{2}\right)$ obtained from Theorems 2.2, 2.3, 4.2 and 4.3 .

Lemma 6.5. For every $v \in L^{2}(\Omega), u \in L^{\infty}(\Omega)$, with $\alpha \leq u \leq \beta, M>0$ and $\lambda \in \mathbb{R}^{n_{e}+n_{i}}$, with $\|\lambda\| \leq M$, there exists $C_{M}>0$ such that

$$
\left|\left[\frac{\partial^{2} \mathcal{L}}{\partial u^{2}}(u, \lambda)-\frac{\partial^{2} \mathcal{L}_{h}}{\partial u_{h}^{2}}(u, \lambda)\right] v^{2}\right| \leq C_{M} h^{\sigma}\|v\|_{L^{2}(\Omega)}^{2}
$$

where $\sigma$ is given as in Lemma 4.4 .

Lemma 6.6. For every $M>0, v \in L^{2}(\Omega), u_{i} \in L^{\infty}(\Omega)$ and $\lambda_{i} \in \mathbb{R}^{n_{e}+n_{i}}$, with $\alpha \leq u_{i} \leq \beta$ and $\left\|\lambda_{i}\right\| \leq M$, $i=1,2$, there exists $C_{M}>0$ such that

$$
\left|\left[\frac{\partial^{2} \mathcal{L}_{h}}{\partial u_{h}^{2}}\left(u_{2}, \lambda_{2}\right)-\frac{\partial^{2} \mathcal{L}_{h}}{\partial u_{h}^{2}}\left(u_{1}, \lambda_{1}\right)\right] v^{2}\right| \leq C_{M}\left(\left\|u_{2}-u_{1}\right\|_{L^{\infty}(\Omega)}+\left\|\lambda_{2}-\lambda_{1}\right\|+h^{\sigma}\right)\|v\|_{L^{2}(\Omega)}^{2}
$$

where $\sigma$ is given as in Lemma 4.4.

Given $u_{h}$ by (6.6) and $\left\{\bar{w}_{h j}\right\}_{j \in I_{0}}$ by Lemma 4.8 , we define $\lambda_{h} \in \mathbb{R}^{n_{e}+n_{i}}$

$$
\lambda_{h i}=\left\{\begin{array}{c}
-\int_{\Omega_{h}}\left[\frac{\partial L}{\partial u}\left(x, y_{h}\left(u_{h}\right), u_{h}\right)+\varphi_{h 0}\left(u_{h}\right)\right] \bar{w}_{h i} \mathrm{~d} x, i \in I_{0} \\
0 \text { otherwise. }
\end{array}\right.
$$

From Remark 3.3, Lemma 4.8 and the fact that

$$
\left\|\bar{u}-u_{h}\right\|_{L^{\infty}(\Omega)} \leq C h\|\bar{u}\|_{W^{1, \infty}(\Omega)}
$$


it follows that

$$
\left\|\bar{\lambda}-\lambda_{h}\right\| \leq C h
$$

Now we set

$$
d_{h}(x)=\frac{\partial L}{\partial u_{h}}\left(x, y_{h}\left(u_{h}\right), u_{h}\right)+\sum_{j \in I_{0}} \lambda_{h j} \varphi_{h j}\left(u_{h}\right)
$$

and

$$
\varphi_{h}\left(u_{h}\right)=\varphi_{h 0}\left(u_{h}\right)+\sum_{j=1}^{n_{e}+n_{i}} \lambda_{h j} \varphi_{h j}\left(u_{h}\right)
$$

with $\varphi_{h 0}\left(u_{h}\right)$ and $\varphi_{h j}\left(u_{h}\right)$ being the solutions of (4.7) and (4.10) respectively corresponding to $u=u_{h}$.

Finally we introduce the function $\zeta_{h} \in U_{h}$ as follows

$$
\zeta_{h \mid T}=\left\{\begin{array}{cl}
{\left[-\frac{1}{|T|} \int_{T} \mathrm{~d}_{h}(x) \mathrm{d} x\right]^{+}} & \text {if } u_{h \mid T}=\alpha \\
-\left[\frac{1}{|T|} \int_{T} \mathrm{~d}_{h}(x) \mathrm{d} x\right]^{+} & \text {if } u_{h \mid T}=\beta \\
-\frac{1}{|T|} \int_{T} \mathrm{~d}_{h}(x) \mathrm{d} x & \text { otherwise. }
\end{array}\right.
$$

Then we have

$$
\left\|\zeta_{h}\right\|_{L^{2}(\Omega)} \leq C h
$$

Taking into account Lemma 4.4 and the estimates (6.22) and (6.23), the proof follows the same lines as the corresponding one of $[1]$.

From the definition of $\lambda_{h}, d_{h}$ and $\zeta_{h}$ we easily deduce that

$$
\frac{\partial \mathcal{L}_{h}}{\partial u_{h}}\left(u_{h}, \lambda_{h}\right)\left(v_{h}-u_{h}\right)+\int_{\Omega_{h}} \zeta_{h}(x)\left(v_{h}(x)-u_{h}(x)\right) \mathrm{d} x \geq 0
$$

for every $v_{h} \in U_{h}$ with $\alpha \leq v_{h} \leq \beta$.

We are ready to prove our first error estimate.

Theorem 6.7. Under the assumptions (A1-A3) and supposing that $\bar{u}$ is a regular local minimum of (P) satisfying the sufficient second order optimality condition (3.13), then the following estimate holds

$$
\left\|\bar{u}-\bar{u}_{h}\right\|_{L^{2}(\Omega)} \leq C h^{\sigma}
$$

where $\sigma$ is given by Lemma 4.4

Proof. Taking $v_{h}=\bar{u}_{h}$ in (6.27) and making the addition with (5.2) we get

$$
\left[\frac{\partial \mathcal{L}_{h}}{\partial u_{h}}\left(u_{h}, \lambda_{h}\right)-\frac{\partial \mathcal{L}_{h}}{\partial u_{h}}\left(\bar{u}_{h}, \bar{\lambda}_{h}\right)\right]\left(\bar{u}_{h}-u_{h}\right)+\int_{\Omega_{h}} \zeta_{h}\left(\bar{u}_{h}-u_{h}\right) \mathrm{d} x \geq 0,
$$


which can be written

$$
\left[\frac{\partial \mathcal{L}_{h}}{\partial u_{h}}\left(u_{h}, \lambda_{h}\right)-\frac{\partial \mathcal{L}_{h}}{\partial u_{h}}\left(\bar{u}_{h}, \bar{\lambda}_{h}\right)\right]\left(u_{h}-\bar{u}_{h}\right) \leq \int_{\Omega_{h}} \zeta_{h}\left(\bar{u}_{h}-u_{h}\right) \mathrm{d} x
$$

We still add a new term

$$
\left[\frac{\partial \mathcal{L}_{h}}{\partial u_{h}}\left(u_{h}, \lambda_{h}\right)-\frac{\partial \mathcal{L}_{h}}{\partial u_{h}}\left(\bar{u}_{h}, \lambda_{h}\right)\right]\left(u_{h}-\bar{u}_{h}\right) \leq \int_{\Omega_{h}} \zeta_{h}\left(\bar{u}_{h}-u_{h}\right) \mathrm{d} x+\left[\frac{\partial \mathcal{L}_{h}}{\partial u_{h}}\left(\bar{u}_{h}, \bar{\lambda}_{h}\right)-\frac{\partial \mathcal{L}_{h}}{\partial u_{h}}\left(\bar{u}_{h}, \lambda_{h}\right)\right]\left(u_{h}-\bar{u}_{h}\right) .
$$

Now using mean value theorem we get for $v_{h}=u_{h}+\theta_{h}\left(\bar{u}_{h}-u_{h}\right)$, with $0<\theta_{h}<1$,

$$
\frac{\partial^{2} \mathcal{L}_{h}}{\partial u_{h}^{2}}\left(v_{h}, \lambda_{h}\right)\left(u_{h}-\bar{u}_{h}\right)^{2} \leq \int_{\Omega_{h}} \zeta_{h}\left(\bar{u}_{h}-u_{h}\right) \mathrm{d} x+\left[\frac{\partial \mathcal{L}_{h}}{\partial u_{h}}\left(\bar{u}_{h}, \bar{\lambda}_{h}\right)-\frac{\partial \mathcal{L}_{h}}{\partial u_{h}}\left(\bar{u}_{h}, \lambda_{h}\right)\right]\left(u_{h}-\bar{u}_{h}\right) .
$$

From here we deduce

$$
\frac{\partial^{2} \mathcal{L}_{h}}{\partial u_{h}^{2}}\left(v_{h}, \lambda_{h}\right)\left(u_{h}-\bar{u}_{h}\right)^{2} \leq\left\|\zeta_{h}\right\|_{L^{2}(\Omega)}\left\|\bar{u}_{h}-u_{h}\right\|_{L^{2}(\Omega)}+\left[\frac{\partial \mathcal{L}_{h}}{\partial u_{h}}\left(\bar{u}_{h}, \bar{\lambda}_{h}\right)-\frac{\partial \mathcal{L}_{h}}{\partial u_{h}}\left(\bar{u}_{h}, \lambda_{h}\right)\right]\left(u_{h}-\bar{u}_{h}\right) .
$$

Taking into account (6.26) it comes

$$
\begin{aligned}
\frac{\partial^{2} \mathcal{L}}{\partial u^{2}}(\bar{u}, \bar{\lambda})\left(u_{h}-\bar{u}_{h}\right)^{2} & +\left[\frac{\partial^{2} \mathcal{L}_{h}}{\partial u_{h}^{2}}(\bar{u}, \bar{\lambda})-\frac{\partial^{2} \mathcal{L}}{\partial u^{2}}(\bar{u}, \bar{\lambda})\right]\left(u_{h}-\bar{u}_{h}\right)^{2} \\
& +\left[\frac{\partial^{2} \mathcal{L}_{h}}{\partial u_{h}^{2}}\left(v_{h}, \lambda_{h}\right)-\frac{\partial^{2} \mathcal{L}_{h}}{\partial u_{h}^{2}}(\bar{u}, \bar{\lambda})\right]\left(u_{h}-\bar{u}_{h}\right)^{2} \leq C h\left\|u_{h}-\bar{u}_{h}\right\|_{L^{2}(\Omega)} \\
& +\left[\frac{\partial \mathcal{L}_{h}}{\partial u_{h}}\left(\bar{u}_{h}, \bar{\lambda}_{h}\right)-\frac{\partial \mathcal{L}_{h}}{\partial u_{h}}\left(\bar{u}_{h}, \lambda_{h}\right)\right]\left(u_{h}-\bar{u}_{h}\right) .
\end{aligned}
$$

Let us estimate each of the three terms of the left hand side. For the first term we use (6.9) as follows

$$
\begin{aligned}
\frac{\partial^{2} \mathcal{L}}{\partial u^{2}}(\bar{u}, \bar{\lambda})\left(u_{h}-\bar{u}_{h}\right)^{2}= & \frac{\partial^{2} \mathcal{L}}{\partial u^{2}}(\bar{u}, \bar{\lambda})\left(\tilde{u}_{h}-\bar{u}_{h}\right)^{2}+\frac{\partial^{2} \mathcal{L}}{\partial u^{2}}(\bar{u}, \bar{\lambda})\left(u_{h}-\tilde{u}_{h}\right)^{2} \\
& +2 \frac{\partial^{2} \mathcal{L}}{\partial u^{2}}(\bar{u}, \bar{\lambda})\left(\tilde{u}_{h}-\bar{u}_{h}\right)\left(u_{h}-\tilde{u}_{h}\right) \geq \bar{\mu}\left\|\tilde{u}_{h}-\bar{u}_{h}\right\|_{L^{2}(\Omega)}^{2} \\
& -C\left(\left\|u_{h}-\tilde{u}_{h}\right\|_{L^{2}(\Omega)}^{2}+\left\|\tilde{u}_{h}-\bar{u}_{h}\right\|_{L^{2}(\Omega)}\left\|u_{h}-\tilde{u}_{h}\right\|_{L^{2}(\Omega)}\right)
\end{aligned}
$$

From (6.10) and (6.22) we obtain the estimate

$$
\left\|u_{h}-\tilde{u}_{h}\right\|_{L^{2}(\Omega)} \leq C\left(h^{\sigma}+\left\|\bar{u}-\bar{u}_{h}\right\|_{L^{2}(\Omega)}^{2}\right)
$$

which along with the previous inequality and Young's inequality lead to

$$
\frac{\partial^{2} \mathcal{L}}{\partial u^{2}}(\bar{u}, \bar{\lambda})\left(u_{h}-\bar{u}_{h}\right)^{2} \geq \frac{\bar{\mu}}{2}\left\|\tilde{u}_{h}-\bar{u}_{h}\right\|_{L^{2}(\Omega)}^{2}-C\left(h^{2 \sigma}+\left\|\bar{u}-\bar{u}_{h}\right\|_{L^{2}(\Omega)}^{4}\right) .
$$

From inequality (6.10) it follows

$$
\left\|\tilde{u}_{h}-\bar{u}_{h}\right\|_{L^{2}(\Omega)} \geq\left\|\bar{u}-\bar{u}_{h}\right\|_{L^{2}(\Omega)}-C\left(h^{\sigma}+\left\|\bar{u}-\bar{u}_{h}\right\|_{L^{2}(\Omega)}^{2}\right) .
$$


Thus we conclude our first estimate

$$
\frac{\partial^{2} \mathcal{L}}{\partial u^{2}}(\bar{u}, \bar{\lambda})\left(u_{h}-\bar{u}_{h}\right)^{2} \geq \frac{\bar{\mu}}{4}\left\|\bar{u}-\bar{u}_{h}\right\|_{L^{2}(\Omega)}^{2}-C\left(h^{2 \sigma}+\left\|\bar{u}-\bar{u}_{h}\right\|_{L^{2}(\Omega)}^{4}\right) .
$$

To get the second estimate we use Lemma 6.5 and the inequality

$$
\left\|u_{h}-\bar{u}_{h}\right\|_{L^{2}(\Omega)} \leq\left\|u_{h}-\bar{u}\right\|_{L^{2}(\Omega)}+\left\|\bar{u}_{h}-\bar{u}\right\|_{L^{2}(\Omega)} \leq C h+\left\|\bar{u}_{h}-\bar{u}\right\|_{L^{2}(\Omega)}
$$

to deduce

$$
\left[\frac{\partial^{2} \mathcal{L}_{h}}{\partial u_{h}^{2}}(\bar{u}, \bar{\lambda})-\frac{\partial^{2} \mathcal{L}}{\partial u^{2}}(\bar{u}, \bar{\lambda})\right]\left(u_{h}-\bar{u}_{h}\right)^{2} \geq-C h^{\sigma}\left(h^{2}+\left\|u_{h}-\bar{u}\right\|_{L^{2}(\Omega)}^{2}\right) .
$$

To obtain the third estimate it is enough to use Lemma 6.6 , equations $(6.22,6.23,6.33)$ and the definition of $v_{h}$ and $\lambda_{h}$

$$
\begin{aligned}
{\left[\frac{\partial^{2} \mathcal{L}_{h}}{\partial u_{h}^{2}}\left(v_{h}, \lambda_{h}\right)-\frac{\partial^{2} \mathcal{L}_{h}}{\partial u_{h}^{2}}(\bar{u}, \bar{\lambda})\right]\left(u_{h}-\bar{u}_{h}\right)^{2} } & \geq-C\left(\left\|v_{h}-\bar{u}\right\|_{L^{\infty}(\Omega)}+\left\|\lambda_{h}-\bar{\lambda}\right\|+h^{\sigma}\right)\left\|u_{h}-\bar{u}_{h}\right\|_{L^{2}(\Omega)}^{2} \\
& \geq-C\left(\left\|\bar{u}_{h}-u_{h}\right\|_{L^{\infty}(\Omega)}+h+h^{\sigma}\right)\left(h^{2}+\left\|u_{h}-\bar{u}\right\|_{L^{2}(\Omega)}^{2}\right)
\end{aligned}
$$

Combining the estimates $(6.29,6.32,6.34)$ and $(6.35)$ and taking into account that $(6.2,6.22)$ and (6.23) imply that

$$
\left\|\bar{u}-\bar{u}_{h}\right\|_{L^{2}(\Omega)} \rightarrow 0 \text { and }\left\|u_{h}-\bar{u}_{h}\right\|_{L^{\infty}(\Omega)} \leq\left\|u_{h}-\bar{u}\right\|_{L^{\infty}(\Omega)}+\left\|\bar{u}-\bar{u}_{h}\right\|_{L^{\infty}(\Omega)} \rightarrow 0
$$

we deduce that for $h$ smaller than a certain $h_{0}>0$ we have

$$
\frac{\bar{\mu}}{8}\left\|\bar{u}-\bar{u}_{h}\right\|_{L^{2}(\Omega)}^{2}-C h^{2 \sigma} \leq C h\left\|u_{h}-\bar{u}_{h}\right\|_{L^{2}(\Omega)}+\left[\frac{\partial \mathcal{L}_{h}}{\partial u_{h}}\left(\bar{u}_{h}, \bar{\lambda}_{h}\right)-\frac{\partial \mathcal{L}_{h}}{\partial u_{h}}\left(\bar{u}_{h}, \lambda_{h}\right)\right]\left(u_{h}-\bar{u}_{h}\right) .
$$

Inserting (6.33) in this inequality it follows from Young's inequality and taking $h_{0}$ small enough that

$$
\begin{aligned}
\frac{\bar{\mu}}{8}\left\|\bar{u}-\bar{u}_{h}\right\|_{L^{2}(\Omega)}^{2}-C h^{2 \sigma} \leq & C h\left(h+\left\|\bar{u}-\bar{u}_{h}\right\|_{L^{2}(\Omega)}\right)+\left[\frac{\partial \mathcal{L}_{h}}{\partial u_{h}}\left(\bar{u}_{h}, \bar{\lambda}_{h}\right)-\frac{\partial \mathcal{L}_{h}}{\partial u_{h}}\left(\bar{u}_{h}, \lambda_{h}\right)\right]\left(u_{h}-\bar{u}_{h}\right) \leq C h^{2} \\
& +\frac{\bar{\mu}}{16}\left\|\bar{u}-\bar{u}_{h}\right\|_{L^{2}(\Omega)}^{2}+\left[\frac{\partial \mathcal{L}_{h}}{\partial u_{h}}\left(\bar{u}_{h}, \bar{\lambda}_{h}\right)-\frac{\partial \mathcal{L}_{h}}{\partial u_{h}}\left(\bar{u}_{h}, \lambda_{h}\right)\right]\left(u_{h}-\bar{u}_{h}\right)
\end{aligned}
$$

or equivalently

$$
\frac{\bar{\mu}}{16}\left\|\bar{u}-\bar{u}_{h}\right\|_{L^{2}(\Omega)}^{2} \leq C h^{2 \sigma}+\left[\frac{\partial \mathcal{L}_{h}}{\partial u_{h}}\left(\bar{u}_{h}, \bar{\lambda}_{h}\right)-\frac{\partial \mathcal{L}_{h}}{\partial u_{h}}\left(\bar{u}_{h}, \lambda_{h}\right)\right]\left(u_{h}-\bar{u}_{h}\right) .
$$

Finally let us estimate the second summand of the right hand side. First of all, from (6.23) and (6.33) we get

$$
\begin{array}{r}
\left|\left[\frac{\partial \mathcal{L}_{h}}{\partial u_{h}}\left(\bar{u}_{h}, \bar{\lambda}_{h}\right)-\frac{\partial \mathcal{L}_{h}}{\partial u_{h}}\left(\bar{u}_{h}, \lambda_{h}\right)\right]\left(u_{h}-\bar{u}_{h}\right)\right| \leq\left|\left[\frac{\partial \mathcal{L}_{h}}{\partial u_{h}}\left(\bar{u}_{h}, \bar{\lambda}\right)-\frac{\partial \mathcal{L}_{h}}{\partial u_{h}}\left(\bar{u}_{h}, \lambda_{h}\right)\right]\left(u_{h}-\bar{u}_{h}\right)\right| \\
+\left|\left[\frac{\partial \mathcal{L}_{h}}{\partial u_{h}}\left(\bar{u}_{h}, \bar{\lambda}_{h}\right)-\frac{\partial \mathcal{L}_{h}}{\partial u_{h}}\left(\bar{u}_{h}, \bar{\lambda}\right)\right]\left(u_{h}-\bar{u}_{h}\right)\right| \leq C h\left(h+\left\|\bar{u}_{h}-\bar{u}\right\|_{L^{2}(\Omega)}\right) \\
+\left|\left[\frac{\partial \mathcal{L}_{h}}{\partial u_{h}}\left(\bar{u}_{h}, \bar{\lambda}_{h}\right)-\frac{\partial \mathcal{L}_{h}}{\partial u_{h}}\left(\bar{u}_{h}, \bar{\lambda}\right)\right]\left(u_{h}-\bar{u}_{h}\right)\right| .
\end{array}
$$


By subtracting (3.8) and (5.6) and using (4.12) and (4.16) we obtain

$$
\left\|\bar{\lambda}-\bar{\lambda}_{h}\right\| \leq C\left(h+\left\|\bar{u}-\bar{u}_{h}\right\|_{L^{2}(\Omega)}\right) .
$$

Using $(4.12,6.10,6.30)$ and $(6.38)$ in the identity

$$
\begin{aligned}
{\left[\frac{\partial \mathcal{L}_{h}}{\partial u_{h}}\left(\bar{u}_{h}, \bar{\lambda}_{h}\right)-\frac{\partial \mathcal{L}_{h}}{\partial u_{h}}\left(\bar{u}_{h}, \bar{\lambda}\right)\right]\left(u_{h}-\bar{u}_{h}\right)=} & \sum_{j=1}^{n_{e}+n_{i}}\left(\bar{\lambda}_{h j}-\bar{\lambda}_{j}\right) G_{h j}^{\prime}\left(\bar{u}_{h}\right)\left(u_{h}-\bar{u}_{h}\right) \\
= & \sum_{j=1}^{n_{e}+n_{i}}\left(\bar{\lambda}_{h j}-\bar{\lambda}_{j}\right)\left\{\left[G_{h j}^{\prime}\left(\bar{u}_{h}\right)-G_{j}^{\prime}(\bar{u})\right]\left(\tilde{u}_{h}-\bar{u}_{h}\right)\right. \\
& \left.+\left[G_{j}^{\prime}(\bar{u})\left(\tilde{u}_{h}-\bar{u}_{h}\right)\right]+\left[G_{h j}^{\prime}\left(\bar{u}_{h}\right)\left(u_{h}-\tilde{u}_{h}\right)\right]\right\}
\end{aligned}
$$

we get

$$
\begin{aligned}
\left|\left[\frac{\partial \mathcal{L}_{h}}{\partial u_{h}}\left(\bar{u}_{h}, \bar{\lambda}_{h}\right)-\frac{\partial \mathcal{L}_{h}}{\partial u_{h}}\left(\bar{u}_{h}, \bar{\lambda}\right)\right]\left(u_{h}-\bar{u}_{h}\right)\right| \leq & C\left\|\bar{\lambda}-\bar{\lambda}_{h}\right\| \sum_{j=1}^{n_{e}+n_{i}}\left\{\left\|\varphi_{h j}\left(\bar{u}_{h}\right)-\varphi_{j \bar{u}}\right\|_{L^{2}(\Omega)}\|\| \tilde{u}_{h}-\bar{u}_{h} \|_{L^{2}(\Omega)}\right. \\
& \left.+\left\|u_{h}-\tilde{u}_{h}\right\|_{L^{2}(\Omega)}\right\}+\sum_{j=1}^{n_{e}+n_{i}}\left|\bar{\lambda}_{j}-\bar{\lambda}_{h j} \| G_{j}^{\prime}(\bar{u})\left(\tilde{u}_{h}-\bar{u}_{h}\right)\right| \\
\leq & C\left(h+\left\|\bar{u}-\bar{u}_{h}\right\|_{L^{2}(\Omega)}\right)\left\{\left(h^{2}+\left\|\bar{u}-\bar{u}_{h}\right\|_{L^{2}(\Omega)}\right)\left(h^{\sigma}+\left\|\bar{u}-\bar{u}_{h}\right\|_{L^{2}(\Omega)}\right)\right. \\
& \left.+\left(h^{\sigma}+\left\|\bar{u}-\bar{u}_{h}\right\|_{L^{2}(\Omega)}^{2}\right)\right\}+\sum_{j=1}^{n_{e}+n_{i}}\left|\bar{\lambda}_{j}-\bar{\lambda}_{h j} \| G_{j}^{\prime}(\bar{u})\left(\tilde{u}_{h}-\bar{u}_{h}\right)\right| \\
\leq & C\left(h+\left\|\bar{u}-\bar{u}_{h}\right\|_{L^{2}(\Omega)}\right)\left(h^{\sigma}+\left\|\bar{u}-\bar{u}_{h}\right\|_{L^{2}(\Omega)}^{2}\right) \\
& +\sum_{j=1}^{n_{e}+n_{i}}\left|\bar{\lambda}_{j}-\bar{\lambda}_{h j} \| G_{j}^{\prime}\left(\bar{u}_{)}\right)\left(\tilde{u}_{h}-\bar{u}_{h}\right)\right| .
\end{aligned}
$$

It remains to estimate the terms $\left|\bar{\lambda}_{j}-\bar{\lambda}_{h j}\right|\left|G_{j}^{\prime}(\bar{u})\left(\tilde{u}_{h}-\bar{u}_{h}\right)\right|$. According to Lemma 6.3, all these terms are zero except for those $j>n_{e}$, such that $G_{j}(\bar{u})=0, \bar{\lambda}_{j}=0$ and $\bar{\lambda}_{h j}>0$. In this case it follows from (5.1) that $G_{h j}\left(\bar{u}_{h}\right)=0$. By using a Taylor development we get

$$
G_{j}\left(\bar{u}_{h}\right)=G_{j}(\bar{u})+G_{j}^{\prime}(\bar{u})\left(\bar{u}_{h}-\bar{u}\right)+\frac{1}{2} G_{j}^{\prime \prime}\left(\bar{v}_{h}\right)\left(\bar{u}_{h}-\bar{u}\right)^{2}
$$

and thanks to (4.13)

$$
\left|G_{j}^{\prime}(\bar{u})\left(\bar{u}_{h}-\bar{u}\right)\right| \leq\left|G_{j}\left(\bar{u}_{h}\right)-G_{h j}\left(\bar{u}_{h}\right)\right|+\left|\frac{1}{2} G_{j}^{\prime \prime}\left(\bar{v}_{h}\right)\left(\bar{u}_{h}-\bar{u}\right)^{2}\right| \leq C\left(h^{\sigma}+\left\|\bar{u}_{h}-\bar{u}\right\|_{L^{2}(\Omega)}^{2}\right) .
$$

Therefore

$$
\begin{aligned}
\left|G_{j}^{\prime}(\bar{u})\left(\tilde{u}_{h}-\bar{u}_{h}\right)\right| \leq\left|G_{j}^{\prime}(\bar{u})\left(\bar{u}-\bar{u}_{h}\right)\right|+\left|G_{j}^{\prime}(\bar{u})\left(\tilde{u}_{h}-\bar{u}\right)\right| & \leq C\left(h^{\sigma}+\left\|\bar{u}_{h}-\bar{u}\right\|_{L^{2}(\Omega)}^{2}+\left\|\tilde{u}_{h}-\bar{u}\right\|_{L^{2}(\Omega)}\right) \\
& \leq C\left(h^{\sigma}+\left\|\bar{u}_{h}-\bar{u}\right\|_{L^{2}(\Omega)}^{2}\right) .
\end{aligned}
$$


Using once again (6.38) it turns out

$$
\sum_{j=1}^{n_{e}+n_{i}}\left|\bar{\lambda}_{j}-\bar{\lambda}_{h j}\right|\left|G_{j}^{\prime}(\bar{u})\left(\tilde{u}_{h}-\bar{u}_{h}\right)\right| \leq C\left(h+\left\|\bar{u}_{h}-\bar{u}\right\|_{L^{2}(\Omega)}\right)\left(h^{\sigma}+\left\|\bar{u}_{h}-\bar{u}\right\|_{L^{2}(\Omega)}^{2}\right) .
$$

Finally $(6.36,6.37)$ and $(6.39)$ along with the above inequality lead to (6.28).

We finish by proving the error estimates for the Lagrange multipliers as well as for the controls in the $L^{\infty}(\Omega)$ norm.

Theorem 6.8. Under the assumptions of Theorem 6.7, then the following estimate holds

$$
\left\|\bar{\lambda}-\bar{\lambda}_{h}\right\|+\left\|\bar{u}-\bar{u}_{h}\right\|_{L^{\infty}(\Omega)} \leq C h^{\sigma}
$$

where $\sigma$ is given by Lemma 4.4

Proof. First of all let us notice that the estimate for the Lagrange multipliers follow from (6.38) and (6.28). Let us derive the error estimates in the $L^{\infty}$ norm for the controls. The following inequalities were stated in the proof of Theorem 6.1

$$
\left\|\bar{u}-\bar{u}_{h}\right\|_{L^{\infty}\left(\Omega_{h}\right)} \leq C\left(h+\left\|\varphi_{\bar{u}}-\varphi_{h}\left(\bar{u}_{h}\right)\right\|_{L^{\infty}\left(\Omega_{h}\right)}+\left\|y_{\bar{u}}-y_{h}\left(\bar{u}_{h}\right)\right\|_{L^{\infty}\left(\Omega_{h}\right)}\right)
$$

and

$$
\left\|\bar{u}-\bar{u}_{h}\right\|_{L^{\infty}\left(\Omega \backslash \Omega_{h}\right)} \leq \bar{c} h+\left\|\bar{u}-\bar{u}_{h}\right\|_{L^{\infty}\left(\Omega_{h}\right)} .
$$

Therefore

$$
\left\|\bar{u}-\bar{u}_{h}\right\|_{L^{\infty}(\Omega)} \leq C\left(h+\left\|\varphi_{\bar{u}}-\varphi_{h}\left(\bar{u}_{h}\right)\right\|_{L^{\infty}\left(\Omega_{h}\right)}+\left\|y_{\bar{u}}-y_{h}\left(\bar{u}_{h}\right)\right\|_{L^{\infty}\left(\Omega_{h}\right)}\right) .
$$

From $(4.13,6.28)$ and the estimates already proved for the Lagrange multipliers we get

$$
\begin{aligned}
\left\|\varphi_{\bar{u}}-\varphi_{h}\left(\bar{u}_{h}\right)\right\|_{L^{\infty}\left(\Omega_{h}\right)} \leq & \left\|\varphi_{0 \bar{u}}-\varphi_{h 0}\left(\bar{u}_{h}\right)\right\|_{L^{\infty}\left(\Omega_{h}\right)}+\sum_{j=1}^{n_{e}+n_{i}}\left\|\bar{\lambda}_{j} \varphi_{j \bar{u}}-\bar{\lambda}_{h j} \bar{\varphi}_{h j}\left(\bar{u}_{h}\right)\right\|_{L^{\infty}\left(\Omega_{h}\right)} \\
\leq & \left\|\varphi_{0 \bar{u}}-\varphi_{h 0}\left(\bar{u}_{h}\right)\right\|_{L^{\infty}\left(\Omega_{h}\right)}+\sum_{j=1}^{n_{e}+n_{i}}\left\{\left|\bar{\lambda}_{j}-\bar{\lambda}_{h j}\right|\left\|\varphi_{j \bar{u}}\right\|_{L^{\infty}\left(\Omega_{h}\right)}\right. \\
& \left.+\left|\bar{\lambda}_{h j}\right|\left\|\varphi_{j \bar{u}}-\bar{\varphi}_{h j}\left(\bar{u}_{h}\right)\right\|_{L^{\infty}\left(\Omega_{h}\right)}\right\} \leq C\left(h^{\sigma}+\left\|\bar{u}-\bar{u}_{h}\right\|_{L^{2}(\Omega)}\right) \leq C h^{\sigma} .
\end{aligned}
$$

Using once again (4.13) we deduce (6.40) from (6.41) and the above inequality.

In the proof of the previous Theorem we have used the estimate

$$
\left\|\bar{y}-\bar{y}_{h}\right\|_{L^{\infty}(\Omega)} \leq C h^{\sigma}
$$

which follows from Lemma 4.4 and (6.28). Above we have also proved that

$$
\left\|\bar{\varphi}-\bar{\varphi}_{h}\right\|_{L^{\infty}(\Omega)}=\left\|\bar{\varphi}-\bar{\varphi}_{h}\right\|_{L^{\infty}\left(\Omega_{h}\right)} \leq C h^{\sigma} .
$$

Now using once again Lemma 4.4 and (6.28) it comes

$$
\left\|\bar{y}-\bar{y}_{h}\right\|_{H^{1}(\Omega)}+\left\|\bar{\varphi}-\bar{\varphi}_{h}\right\|_{H^{1}(\Omega)} \leq C h^{\sigma} .
$$


Finally let me say that the error estimates seem to be optimal in the cases where $\sigma=1$. This opinion is based on the fact that the interpolation error of functions of $C^{0,1}(\bar{\Omega})$ by piecewise constant functions is of order $h$. For $\sigma=1 / 2$ we do not know if the estimates can be improved. The difficulty appears when studying the $L^{\infty}$ error estimates of the approximations of the state equations by finite element methods; see Casas and Mateos [6].

\section{REFERENCES}

[1] N. Arada, E. Casas and F. Tröltzsch, Error estimates for the numerical approximation of a semilinear elliptic control problem. Comp. Optim. Appl. (to appear).

[2] V. Arnautu and P. Neittaanmäki, Discretization estimates for an elliptic control problem. Numer. Funct. Anal. Optim. (1998) 431-464.

[3] J. Bonnans and E. Casas, Contrôle de systèmes elliptiques semilinéaires comportant des contraintes sur l'état, in Nonlinear Partial Differential Equations and Their Applications, Vol. 8, Collège de France Seminar, edited by H. Brezis and J. Lions. Longman Scientific \& Technical, New York (1988) 69-86.

[4] J. Bonnans and H. Zidani, Optimal control problems with partially polyhedric constraints. SIAM J. Control Optim. 37 (1999) 1726-1741.

[5] E. Casas and M. Mateos, Second order optimality conditions for semilinear elliptic control problems with finitely many state constraints. SIAM J. Control Optim. 40 (2002) 1431-1454.

[6] - Uniform convergence of the fem. applications to state constrained control problems. Comp. Appl. Math. 21 (2002).

[7] E. Casas, M. Mateos and L. Fernández, Second-order optimality conditions for semilinear elliptic control problems with constraints on the gradient of the state. Control Cybernet. 28 (1999) 463-479.

[8] E. Casas and F. Tröltzsch, Second order necessary optimality conditions for some state-constrained control problems of semilinear elliptic equations. App. Math. Optim. 39 (1999) 211-227.

[9] - Second order necessary and sufficient optimality conditions for optimization problems and applications to control theory. SIAM J. Optim. (to appear).

[10] E. Casas, F. Tröltzsch and A. Unger, Second order sufficient optimality conditions for a nonlinear elliptic control problem. J. Anal. Appl. 15 (1996) 687-707.

[11] Second order sufficient optimality conditions for some state-constrained control problems of semilinear elliptic equations. SIAM J. Control Optim. 38 (2000) 1369-1391.

[12] P. Ciarlet, The Finite Element Method for Elliptic Problems. North-Holland, Amsterdam (1978).

[13] F. Clarke, A new approach to Lagrange multipliers. Math. Oper. Res. 1 (1976) 165-174.

[14] R. Falk, Approximation of a class of optimal control problems with order of convergence estimates. J. Math. Anal. Appl. 44 (1973) 28-47.

[15] T. Geveci, On the approximation of the solution of an optimal control problem governed by an elliptic equation. RAIRO: Numer. Anal. 13 (1979) 313-328.

[16] H. Goldberg and F. Tröltzsch, Second order sufficient optimality conditions for a class of nonlinear parabolic boundary control problems. SIAM J. Control Optim. 31 (1993) 1007-1025.

[17] P. Grisvard, Elliptic Problems in Nonsmooth Domains. Pitman, Boston-London-Melbourne (1985).

[18] K. Malanowski, C. Büskens and H. Maurer, Convergence of approximations to nonlinear control problems, in Mathematical Programming with Data Perturbation, edited by A. Fiacco. New York, Marcel Dekker, Inc. (1997) 253-284.

[19] M. Mateos, Problemas de control óptimo gobernados por ecuaciones semilineales con restricciones de tipo integral sobre el gradiente del estado, Ph.D. Thesis. University of Cantabria (2000).

[20] P. Raviart and J. Thomas, Introduction à L'analyse Numérique des Equations aux Dérivées Partielles. Masson, Paris (1983).

[21] J. Raymond and F. Tröltzsch, Second order sufficient optimality conditions for nonlinear parabolic control problems with state-constraints. Discrete Contin. Dynam. Systems 6 (2000) 431-450. 